\title{
Coherent Atom-Phonon Interaction through Mode Field Coupling in Hybrid Optomechanical Systems
}

\author{
Michele Cotrufo* and Andrea Fiore \\ Department of Applied Physics, Eindhoven University of Technology, 5600 MB Eindhoven, The Netherlands \\ Ewold Verhagen \\ Center for Nanophotonics, AMOLF, Science Park 104, 1098 XG Amsterdam, The Netherlands
}

(Received 13 October 2016; published 30 March 2017)

\begin{abstract}
We propose a novel type of optomechanical coupling which enables a tripartite interaction between a quantum emitter, an optical mode, and a macroscopic mechanical oscillator. The interaction uses a mechanism we term mode field coupling: a mechanical displacement modifies the spatial distribution of the optical mode field, which, in turn, modulates the emitter-photon coupling rate. In properly designed multimode optomechanical systems, we can achieve situations in which mode field coupling is the only possible interaction pathway for the system. This enables, for example, swapping of a single excitation between emitter and phonon, creation of nonclassical states of motion, and mechanical ground-state cooling in the bad-cavity regime. Importantly, the emitter-phonon coupling rate can be enhanced through an optical drive field, allowing active control of the emitter-phonon coupling for realistic experimental parameters.
\end{abstract}

DOI: 10.1103/PhysRevLett.118.133603

Interfacing different quantum systems, such as atoms, photons, and phonons, is a key requirement for quantum information processing. The well-established framework of cavity quantum electrodynamics (CQED) interfaces photons-ideal for communication-to natural or artifical atoms (quantum emitters, QEs), whose strong nonlinearities enable quantum processing. Mechanical resonators have recently come to the forefront due to their large coherence times and their interaction with photons in cavity optomechanical systems [1]. Moreover, creating nonclassical states in macroscopic mechanical systems is appealing for fundamental studies of quantum physics $[2,3]$. In these contexts, establishing an efficient and controllable interaction between phonons and QEs would be highly beneficial as it would enable using the QE nonlinearity for the creation and manipulation of phononic quantum states [4].

Different approaches have been proposed to realize such an interaction. First, a phonon can directly couple to a solidstate QE through mechanical strain [5-8]. Large coupling rates can be obtained in specific systems, but this effect is difficult to engineer and to dynamically control. A second approach couples mechanical modes dispersively to an optical cavity, which, in turn, interacts with a QE [9-12]. Tripartite entanglement and atom-assisted optomechanical cooling are predicted in the so-far-elusive regimes when the optomechanical interaction is nonlinear at the quantum level [9] or when the emitter-field coupling rate approaches the emitter frequency [11]. Additionally, a QE-phonon interaction occurs in molecules and solids when the electronic and vibrational degrees of freedom are coupled, leading to inelastic scattering processes. Natural Raman transitions have been used to transfer a photon's quantum state to an optical phonon in diamond [13,14], but the extremely high frequency and large dissipation limit general applications for quantum processing.

In this Letter, we propose a novel optomechanical effect that provides an explicit, engineerable, and optically controllable interaction between a $\mathrm{QE}$ and a macroscopic mechanical oscillator. The interaction arises from a mechanically induced modification of the spatial distribution of the optical field [Fig. 1(a)], which, in turn, modulates the QE-photon coupling rate. We show that this interaction is particularly strong in systems of weakly interacting optical cavities because the field variation, upon a perturbation, scales inversely with the frequency spacing between optical modes. In such systems, the combined effect of different bipartite interactions gives rise to a novel QE-photon-phonon coupling that we term mode field coupling (MFC). We show that MFC can become the only allowed interaction, enabling, e.g., QE-phonon excitation swapping and mechanical ground-state cooling in the badcavity regime. Importantly, the interaction can be controlled and enhanced by the optical field intensity, resulting in optically controlled emitter-phonon coherent manipulation. This coupling, and the resulting Hamiltonian, share important traits with Raman-like processes in trapped ions [15], which have proven powerful in controlling the motional state of single ions. MFC has, however, two distinct features: it involves large-mass macroscopic resonators, and its rate is nonetheless large enough to overcome the large decoherence typical of solid-state QEs.

Model.-We consider a standard CQED setup, in which a two-level QE couples to an optical cavity mode through the Hamiltonian $\hat{H}=\omega_{A} \hat{\sigma}_{z} / 2+\omega_{c} \hat{a}^{\dagger} \hat{a}+g\left(\hat{a} \hat{\sigma}_{+}+\hat{a}^{\dagger} \hat{\sigma}_{-}\right)$, 


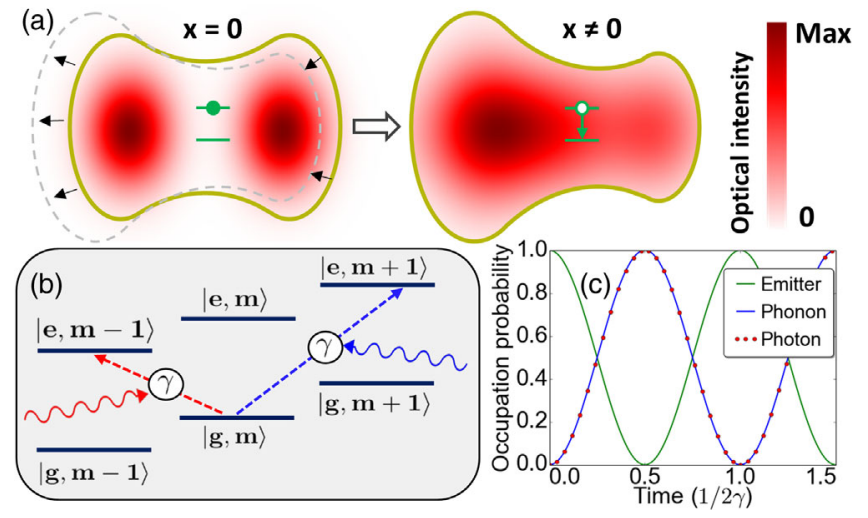

FIG. 1. (a) Sketch of the proposed concept. An optical cavity (yellow solid line) defines an electric field (red pattern), which is initially (left) zero at the QE position (green symbol). Upon boundary displacement (right), the field seen by the QE becomes nonzero and radiative transitions can occur. (b) Phononnonconserving transitions achievable through MFC. A photon red detuned (blue detuned) by $\Omega_{M}$ with respect to the QE stimulates transitions from $|g, m\rangle$ to $|e, m-1\rangle(|e, m+1\rangle)$. (c) Time evolution dictated by the Hamiltonian in Eq. (1), with the QE initially excited and $\omega_{c}=\omega_{A}-\Omega_{M}$.

where $\omega_{A}\left(\omega_{c}\right)$ denotes the $\mathrm{QE}$ (optical) frequency, $\hat{a}$ is the photon annihilation operator, $\hat{\sigma}_{ \pm, z}$ are Pauli operators describing the $\mathrm{QE}$, and $\hbar=1$. We initially neglect any loss, focusing on conservative interactions. The QE-photon coupling rate $g=-\mathbf{d} \cdot \mathcal{E}_{0}$ is determined by the emitter's transition dipole moment $\mathbf{d}$ and the electric field per photon $\mathcal{E}_{0}$ of the optical mode at the emitter position. Next, we consider a mechanical oscillator with frequency $\Omega_{M}$ and phonon annihilation operator $\hat{b}$. In a standard dispersively coupled optomechanical system, the resonator's displacement $\hat{x}=x_{\mathrm{zpf}}\left(\hat{b}+\hat{b}^{\dagger}\right)$ affects the cavity frequency. This interaction is quantified by the coupling rate $g_{0}=$ $-\left(\partial \omega_{c} / \partial x\right) x_{\mathrm{zpf}}$, where $x_{\mathrm{zpf}}$ is the zero-point motion amplitude. Here, we consider a fundamentally different situation in which the mechanical displacement induces a variation of the spatial distribution of the cavity field [Fig. 1(a)], while $\omega_{c}$ is negligibly affected. As a direct consequence, the emitter-cavity coupling rate $g$ becomes dependent on the mechanical position. Up to first order in $\hat{x}, g(\hat{x})=$ $g(0)+\gamma\left(\hat{b}+\hat{b}^{\dagger}\right)$, where we defined the MFC coupling rate $\gamma=\left.(\partial g / \partial x)\right|_{x=0} x_{\mathrm{zpf}}$. Inserting this expression in the Hamiltonian leads to the appearance of a tripartite interaction between the QE, optical field, and mechanical resonator. In the specific case that at mechanical equilibrium the field at the emitter's position vanishes [Fig. 1(a)], $g(0)=0$, and the only possible interaction channel is the tripartite one. The interaction Hamiltonian reads

$$
\hat{H}_{\text {int }}=\gamma\left(\hat{b}+\hat{b}^{\dagger}\right)\left(\hat{a} \hat{\sigma}_{+}+\hat{a}^{\dagger} \sigma_{-}\right) .
$$

This Hamiltonian allows swapping the excitation between the three quantum systems under particular resonant conditions. For $\omega_{c} \approx \omega_{A}+\Omega_{M}\left(\omega_{c} \approx \omega_{A}-\Omega_{M}\right)$, the dominant term is $\hat{b}^{\dagger} \hat{\sigma}_{+} \hat{a}+$ H.c. $\left(\hat{b} \hat{\sigma}_{+} \hat{a}+\right.$ H.c. $)$, describing phonon and QE excitation upon photon annihilation (QE excitation due to photon and phonon annihilation) and the reverse process. Depending on the photon energy, therefore, the transitions $|g, m\rangle \leftrightarrow|e, m \pm 1\rangle$ are realized [Fig. 1(b)], where $e(g)$ denotes the $\mathrm{QE}$ excited (ground) state and $m$ the phonon number. Figure 1(c) shows the lossless time evolution described by Eq. (1) for $\omega_{c}=\omega_{A}-\Omega_{M}$, with only the QE initially excited. The excitation oscillates at a frequency $2 \gamma$ between the QE and the state formed by one photon and one phonon. Next, we consider pumping the cavity with a large coherent field to an average photon number $n_{\text {cav }}$, writing the cavity field as $\hat{a}=\sqrt{n_{\text {cav }}}+\delta \hat{a}$. Neglecting for now the fluctuations $\delta \hat{a}$ (valid for $n_{\text {cav }} \gg 1$ ), the Hamiltonian reads

$$
\hat{H}_{\mathrm{int}}=\gamma \sqrt{n_{\text {cav }}}\left(\hat{b}+\hat{b}^{\dagger}\right)\left(\hat{\sigma}_{+}+\sigma_{-}\right),
$$

which describes a coherent QE-phonon interaction, with a coupling rate controlled by $n_{\text {cav }}$. Thus, the optical intensity can enhance the QE-phonon coupling and, in particular, overcome system losses.

Creating large field variations.-We will now show that the effective MFC interaction Hamiltonian [Eq. (1)] can emerge from standard radiation-pressure forces in coupledcavity systems. As the mode field is the solution of an eigenvalue problem [16], we look for a mechanical perturbation that induces strong changes of the eigenvector without affecting the eigenvalue. This is maximized for quasidegenerate unperturbed eigenvalues, which can be obtained by coupling multiple cavities such that hybridized modes ("supermodes") with well-defined symmetry are formed. Near a symmetry point, i.e., an anticrossing, an odd perturbation breaks symmetry. This localizes the supermodes in one of the cavities, resulting in a large variation of the local mode field.

Indeed, we find an example of MFC [Fig. 2(a)] in two identical optical cavities coupled with rate $J$ (a membranein-the-middle setup $[17,18])$. A mechanical displacement that induces opposite detuning $\pm \Delta$ to each cavity affects the spatial distribution of the supermode amplitudes, and thereby their coupling rate $\left(g^{( \pm)}\right)$with an emitter placed in one cavity [Fig. 2(c)]. For $\Delta / J \ll 1$, the supermode frequencies are constant [Fig. 2(b)]; i.e., dispersive coupling is absent. Importantly, the MFC coupling rate $\gamma$ is enhanced for weak intercavity coupling as it scales with $J^{-1}$ (see Supplemental Material [19]): for weakly interacting cavities $(J \rightarrow 0)$, small deviations from the condition $\Delta=0$ quickly lead to localization of the supermodes into the individual cavities. In the two-cavity system, however, the tripartite MFC interaction competes with the Rabi emitter-photon interaction as $g^{( \pm)}(0) \neq 0$ [Fig. 2(c)].

This direct QE-photon interaction can be suppressed by introducing an additional optical cavity with identical frequency [Fig. 2(d)]. The middle cavity $(T)$, containing the $\mathrm{QE}$, interacts with both lateral cavities with rate $J$, leading to the formation of three supermodes $\hat{a}_{+}, \hat{a}_{-}$, and $\hat{a}_{0}$ $[26,27]$. For zero detuning, the mode $\hat{a}_{0}$ has opposite fields in the lateral cavities and zero field in $T$ [blue line in 
(a)

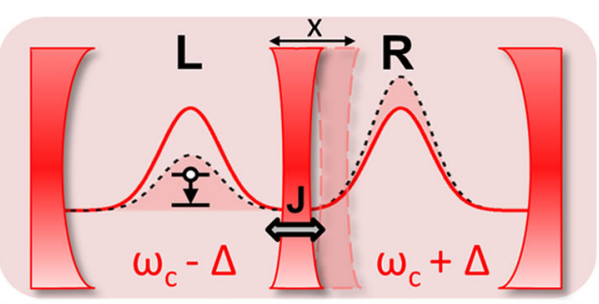

(d)

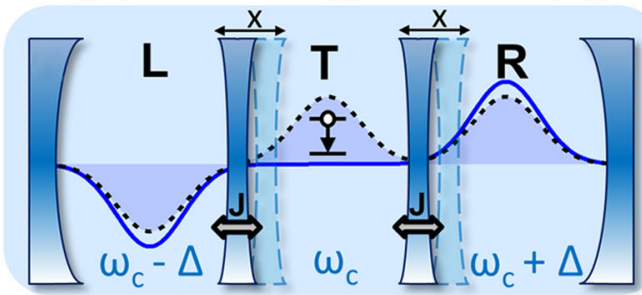

(b)

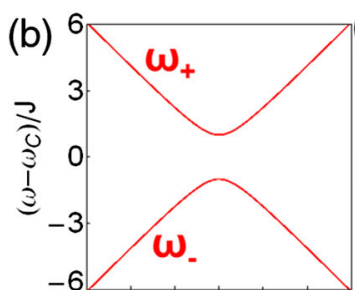

(e)
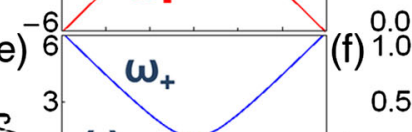
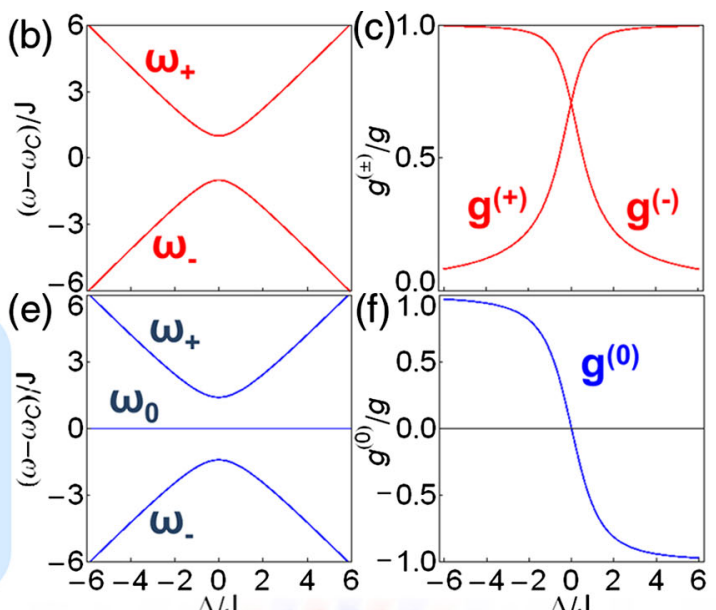

$\Delta / \mathrm{J}$

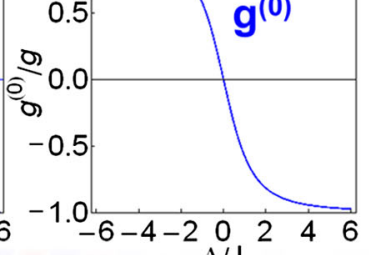

(g)

(h)
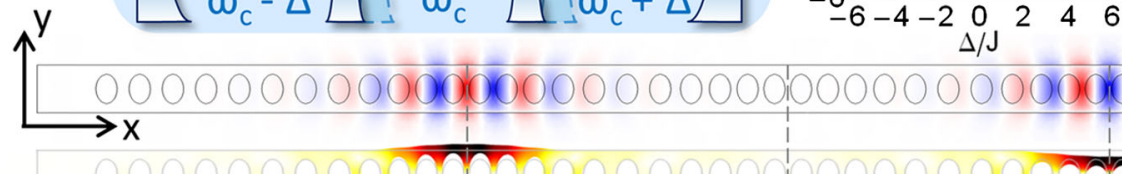

0000000000000000000000000000000000000000000000

FIG. 2. (a) Two identical cavities interact at rate $J$ through a partly transparent movable mirror. The symmetric optical supermode is sketched for zero detuning (red line) and equal and opposite detuning $\pm \Delta$ on the cavities (black dashed line). (b) Supermode frequencies versus $\Delta / J$. (c) Coupling rates between a QE located in the left cavity $(L)$ and the two supermodes (normalized to the coupling rate $g$ with the uncoupled mode in $L$ ) versus $\Delta / J$. (d) Three-cavity system. The field of the supermode of interest $\left(\hat{a}_{0}\right)$ is shown as a blue (black dashed) line for zero $( \pm \Delta)$ detuning of the lateral cavities. (e) Supermode frequencies in the three-cavity system. (f) Normalized coupling rate between a QE located in cavity $T$ and the supermode $\hat{a}_{0}$. (g)-(i) Implementation based on three defect cavities in a photonic crystal nanobeam. Vertical dashed lines mark cavity positions. (g) Electric field ( $y$ component) of $\hat{a}_{0}$ at mechanical equilibrium. (h) Displacement pattern of the selected mechanical mode. (i) Expected field of $\hat{a}_{0}$ upon mechanically induced perturbation $(\Delta / J=0.5)$. Additional details in [19].

Fig. 2(d)], and therefore, does not interact with the QE. We now consider a mechanical mode that detunes only the frequencies of the lateral cavities by $\pm \Delta$, while leaving $T$ unperturbed. This could be realized for example by rigidly connecting the two membranes. More generally, it can be obtained by dispersively coupling each optical cavity at a rate $g_{0}$ to a separate mechanical oscillator [19]. If these three identical oscillators are coupled mechanically, one resulting mechanical supermode has equal and opposite dispersive interaction with the lateral cavities with a rate $\pm g_{0} / \sqrt{2}$ and zero interaction with $T$. The frequency of $\hat{a}_{0}$ is unaltered by such detuning [Fig. 2(e)], while its field in $T$ becomes finite [Fig. 2(d), dashed line], which translates in a large modulation of the coupling rate $g^{(0)}$ between $\hat{a}_{0}$ and the $\mathrm{QE}$ around the value $g^{(0)}=0$ [Fig. 2(f)]. Therefore, the interaction between the emitter, the mode $\hat{a}_{0}$, and the selected mechanical mode will be described by the Hamiltonian in Eq. (1).

Figures 2(g)-2(h) show an implementation of this model in a photonic crystal nanobeam. Cavities are defined by local periodicity variations, which yield colocalized and dispersively coupled optical and mechanical resonances [28,29]. Placing three defect cavities along one nanobeam leads to both optical and mechanical hybridization. The intercavity separation controls the optical interaction rate $J$. The electric field of $\hat{a}_{0}$ [Fig. $\left.2(\mathrm{~g})\right]$ is zero in the central cavity when the mechanical mode is at rest. Figure 2(h) shows the mechanical mode that provides the required detuning on the lateral cavities, which causes $\hat{a}_{0}$ to acquire a finite electric field in the central cavity [Fig. 2(i)].

Full model and numerical calculations.-We now analyze the three-cavity system in detail and show that it behaves as predicted by Eq. (1). For simplicity, we consider only one of the hybridized mechanical supermodes, described by the operator $\hat{b}$, frequency $\Omega_{M}$, and dispersively coupled to the lateral cavities at a rate $\pm g_{0} / \sqrt{2}$. This approach is justified as long as the mechanical mode coupling exceeds the mechanical dissipation [19]. In a frame rotating at $\omega_{c}$, the Hamiltonian is

$$
\begin{aligned}
\hat{H}= & -\hat{\Delta} \hat{a}_{L}^{\dagger} \hat{a}_{L}+\hat{\Delta} \hat{a}_{R}^{\dagger} \hat{a}_{R}+\Omega_{M} \hat{b}^{\dagger} \hat{b}+\frac{\omega_{A}-\omega_{c}}{2} \hat{\sigma}_{z} \\
& +J\left[\hat{a}_{T}^{\dagger}\left(\hat{a}_{L}+\hat{a}_{R}\right)+\text { H.c. }\right]+g\left(\hat{a}_{T} \hat{\sigma}_{+}+\text {H.c. }\right),
\end{aligned}
$$

where we defined $\hat{\Delta}=g_{0} / \sqrt{2}\left(\hat{b}+\hat{b}^{\dagger}\right)$. The first two terms describe the mechanically induced detuning on the lateral cavities. The second row describes optical mode coupling and the Rabi interaction between emitter and cavity $T$. For $J \gg \Omega_{M}$, we can treat $\hat{\Delta}$ quasistatically [18] and diagonalize the optical part of the Hamiltonian by introducing three optical supermodes, $\hat{a}_{ \pm}$and $\hat{a}_{0}$. Up to the first order in $\hat{\Delta} / J$ (generally applicable until very large phonon numbers [19]), we obtain an effective interaction Hamiltonian 
$\hat{H}_{\text {int }}=\gamma\left(\hat{b}+\hat{b}^{\dagger}\right)\left(\hat{a}_{0} \hat{\sigma}_{+}+\right.$H.c. $)+g^{\prime}\left[\left(\hat{a}_{+}-\hat{a}_{-}\right) \hat{\sigma}_{+}+\right.$H.c. $]$.

The first term of Eq. (4) shows the tripartite interaction explicitly, with $\gamma=g g_{0} / 2 J$. The last term describes a Rabi interaction between the emitter and the supermodes $\hat{a}_{ \pm}$, with coupling rate $g^{\prime}=g / \sqrt{2}$. A pure tripartite interaction can therefore be obtained for significant supermode separation $\left(J \gg \Omega_{M}\right)$ and tripartite resonance $\left(\omega_{A} \approx \omega_{c} \pm \Omega_{M}\right)$. Additionally, to let the emitter interact with the supermodes (and not the uncoupled modes), we require $J \gg g$. To verify that the predicted coherent emitter-phonon interaction occurs in a realistic scenario, we numerically calculate the dynamics dictated by the full Hamiltonian in Eq. (3) [19,30]. Out of the many possible systems, we consider the structure of Figs. 2(g)-2(h) made in diamond with a nitrogen vacancy (NV) center as an emitter. The simulated parameters for this system are $\left\{\omega_{c}, \Omega_{M}, g, g_{0}\right\}=2 \pi\left\{4.7 \times 10^{5}, 14,20\right.$, $0.004\}$ GHz. We consider $\omega_{A}=\omega_{c}+\Omega_{M}$ and $J=18 g$, corresponding to single-period cavity separation [19]. The lossless evolution of the three-cavity system \{ shown in Fig. S(3)a of [19]\} agrees perfectly with that of the MFC Hamiltonian [Fig. 1(c)] and verifies the predicted QEphonon oscillation period $\pi / \gamma=4.5 \mu \mathrm{s}$. This confirms that for realistic choices of parameters, a purely tripartite interaction is obtained in the three-cavity system. In order to overcome losses, unavoidable in an experimental setting, the coupling rate can be enhanced by selectively pumping the supermode $\hat{a}_{0}$ [19]. Figure 3(a) shows the evolution of the three-cavity system [Eq. (3)], with dissipations introduced through Lindblad operators [19], using cavity decay rate $\kappa / 2 \pi=3 \mathrm{GHz}$ and emitter decay rate $\Gamma / 2 \pi=0.05 \mathrm{GHz}$
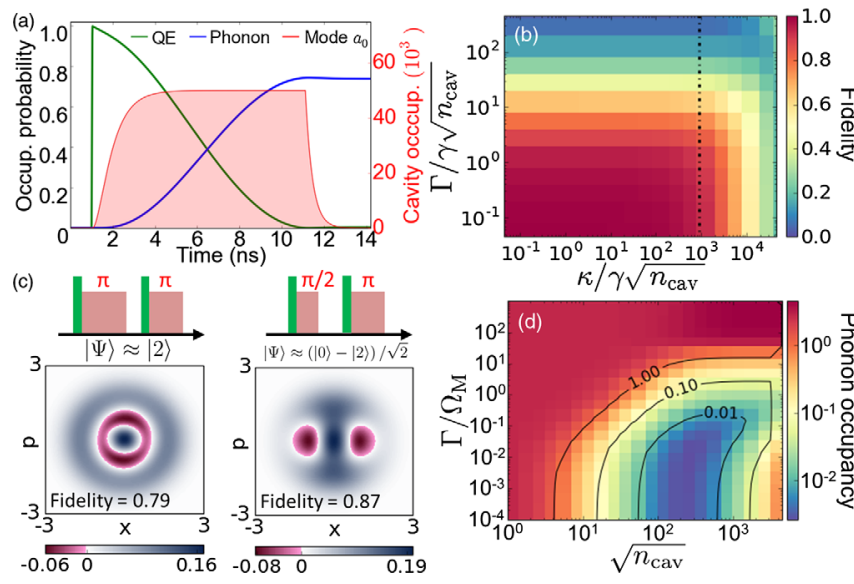

FIG. 3. Numerical calculations based on the three-cavity Hamiltonian [Eq. (3), parameters in text]. (a) The QE is excited at $T=1 \mathrm{~ns}$ and subsequently the mode $\hat{a}_{0}$ (red shaded area) is strongly pumped. (b) Fidelity of the created one-phonon state versus $\Gamma$ and $\kappa$ for $n_{\text {cav }}=5 \times 10^{4}$. The vertical dashed-dotted line indicates $\kappa=2 g_{0} J \sqrt{n_{\text {cav }}} / g$. (c) Two examples of nonclassical mechanical states obtainable. Top plots: pumping sequences. Bottom plots: resultant Wigner maps of the mechanical resonator. (d) Cooling through MFC. Steady-state mean phonon number versus $\Gamma$ and $n_{\text {cav }}$ for continuous optical pumping and $\kappa=10 \Omega_{M}$. (measured for NV centers in photonic crystals [20]). The QE is excited by a short $\pi$ pulse, followed by a pulse that excites $\hat{a}_{0}$ to a maximum population of $n_{\text {cav }}=5 \times 10^{4}$, shown to be experimentally feasible in diamond [29]. We note that the emitter is not directly affected by the large optical intensity as the field is zero at its position. During the optical pulse, the mechanical mode interacts with the QE with a coupling rate $\gamma \sqrt{n_{\text {cav }}}$. After a time $\Delta t=\pi / 2 \gamma \sqrt{n_{\text {cav }}}$, the pump is switched off, suppressing the interaction and leaving the system in a one-phonon state with a fidelity of 0.86 . Its nonclassical nature is emphasized by the characteristic negativity of the Wigner function (shown in Fig. S(3)b of [19]). The fidelity of the onephonon state creation can be made arbitrarily close to one [Fig. 3(b)] by reducing the QE decay rate (so that $\Gamma \ll \gamma \sqrt{n_{\text {cav }}}$ ) and the optical losses $\kappa$ of the supermodes $\hat{a}_{ \pm}$, which introduce additional decay for the QE due to the finite optical linewidth. This decay is negligible when $\kappa \ll$ $2 g_{0} J \sqrt{n_{\text {cav }}} / g$ [dashed-dotted line in Fig. 3(b)] [19]. The influence of $n_{\text {cav }}$ and QE dephasing on the fidelity is discussed in the Supplemental Material [19].

The optical control of the QE-phonon interaction allows creating also other nonclassical mechanical states by pumping the $\mathrm{QE}$ and the optical mode with properly controlled pulse sequences. As an example, we demonstrate the preparation of a Fock state $|\Psi\rangle=|2\rangle$ and a superposition state $|\Psi\rangle=(|0\rangle-|2\rangle) / \sqrt{2}$ using the same parameters as Fig. 3(a). The QE is excited twice, each time followed by either a $\pi$ - or a $\pi / 2$-pulse on the optical mode. Figure 3(c) shows the resultant Wigner maps, displaying the expected nonclassical signatures for these states, which are prepared with fidelities 0.79 and 0.87 , respectively. Interestingly, this interaction could also be employed to reconstruct the density matrix of mechanical states with methods analogous to trapped-ion experiments [15]. Alternative methods to characterize the produced states involve tomography using the dispersive interaction of the mechanical supermode with a different cavity mode [1].

The proposed QE-phonon interaction can also be used to cool the mechanical resonator to its ground state. The cooling cycle is triggered by a red-detuned cavity photon, which excites the QE upon annihilation of a phonon. The excitation is subsequently dissipated through the QE decay. Differently from standard optomechanical cooling [1], this mechanism can achieve ground-state cooling in the bad cavity regime $\left(\kappa \gg \Omega_{M}\right.$ ), while resolved sideband operation is required only for the $\mathrm{QE}\left(\Gamma<\Omega_{M}\right)$. Figure 3(d) shows the steady-state phonon population in the three-cavity system versus $\Gamma$ and $n_{\text {cav }}$ for $\kappa=10 \Omega_{M}$, finite mechanical losses $\left(\Gamma_{M} / 2 \pi=\right.$ $50 \mathrm{kHz}$ ), and thermal initial phonon occupation $n_{\mathrm{th}}=4$. As expected, ground-state cooling is possible for $\Gamma / \Omega_{M} \lesssim 1$. Phonon population lower than 0.1 can be achieved with $n_{\text {cav }} \lesssim 10^{3}$ and realistic QE decay rates. The phonon population increase for large $n_{\text {cav }}$ is attributed to the onset of ultrastrong coupling, as $\gamma \sqrt{n_{\text {cav }}}$ approaches $\Omega_{M}$. For small $\Gamma$, the QE total decay rate is dominated by the additional emission into the supermodes $\hat{a}_{ \pm}$(which read 
$\Gamma^{( \pm)}=g^{2} \kappa / 4 J^{2} \approx 2 \pi \times 0.11 \mathrm{GHz}$ [19]), which explains the saturation of the phonon population for $\Gamma / \Omega_{M}<10^{-2}$.

In conclusion, we have introduced a new kind of emitterphoton-phonon interaction in hybrid-optomechanical systems, based on mechanically induced variations of the electric field spatial pattern. The interaction is particularly strong in weakly interacting multicavity systems as it scales inversely with the intercavity coupling rate $J$. For large optical drives, this mechanism leads to an emitter-phonon coherent interaction whose strength is controlled by the optical intensity. Emitter-phonon excitation swapping and mechanical groundstate cooling are possible with feasible experimental parameters. The proposed interaction strength is much larger than effects obtainable in single-mode systems, which require the ultrastrong coupling regime $\left(g \approx \omega_{c}\right)$ to have comparable rates [10-12,19]. Differently from strain-based methods [5-8], the proposed mechanism is not limited to a specific choice of emitters and material systems, and it could even be applied to atoms trapped near a mechanical resonator [31-33]. Moreover, it provides strong quantum nonlinearities without requiring the single-photon strong optomechanical coupling regime $\left(g_{0} \gg \kappa\right)$. In perspective, the proposed optically controlled emitter-phonon interaction paves the way for, e.g., control of spontaneous phonon emission, creation of nonclassical states of motion, and phonon lasing.

The authors acknowledge L. Midolo for first pointing out the possibility of displacement-induced field variations in optomechanical cavities. This work is part of the research programme of the Netherlands Organisation for Scientific Research (NWO). E. V. acknowledges an NWO-Vidi grant for financial support.

*m.cotrufo@tue.nl

[1] M. Aspelmeyer, T. J. Kippenberg, and F. Marquardt, Rev. Mod. Phys. 86, 1391 (2014).

[2] A. D. O'Connell, M. Hofheinz, M. Ansmann, R. C. Bialczak, M. Lenander, E. Lucero, M. Neeley, D. Sank, H. Wang, M. Weides, J. Wenner, J. Martinis, and A. N. Cleland, Nature (London) 464, 697 (2010).

[3] F. Lecocq, J. D. Teufel, J. Aumentado, and R. W. Simmonds, Nat. Phys. 11, 635 (2015).

[4] J.-M. Pirkkalainen, S. Cho, F. Massel, J. Tuorila, T. Heikkilä, P. Hakonen, and M. Sillanpää, Nat. Commun. 6, 6981 (2015).

[5] I. Wilson-Rae, P. Zoller, and A. Imamoglu, Phys. Rev. Lett. 92, 075507 (2004).

[6] T. Ramos, V. Sudhir, K. Stannigel, P. Zoller, and T. J. Kippenberg, Phys. Rev. Lett. 110, 193602 (2013).

[7] D. A. Golter, T. Oo, M. Amezcua, K. A. Stewart, and H. Wang, Phys. Rev. Lett. 116, 143602 (2016).

[8] M. Munsch, A. V. Kuhlmann, D. Cadeddu, J.-M. Gérard, J. Claudon, M. Poggio, and R. J. Warburton, arXiv:1608.03082.

[9] J. Restrepo, C. Ciuti, and I. Favero, Phys. Rev. Lett. 112, 013601 (2014).

[10] S. Barzanjeh, M. H. Naderi, and M. Soltanolkotabi, Phys. Rev. A 84, 063850 (2011).

[11] Y. Chang, H. Ian, and C. Sun, J. Phys. B 42, 215502 (2009).
[12] W. Wang, L. Wang, and H. Sun, J. Korean Phys. Soc. 57, 704 (2010).

[13] K. A. Fisher, D. G. England, J.-P. W. MacLean, P. J. Bustard, K. J. Resch, and B. J. Sussman, Nat. Commun. 7, 11200 (2016).

[14] K. Lee, B. Sussman, M. Sprague, P. Michelberger, K. Reim, J. Nunn, N. Langford, P. Bustard, D. Jaksch, and I. Walmsley, Nat. Photonics 6, 41 (2012).

[15] D. Leibfried, R. Blatt, C. Monroe, and D. Wineland, Rev. Mod. Phys. 75, 281 (2003).

[16] J. D. Joannopoulos, S. G. Johnson, J. N. Winn, and R. D. Meade, Photonic Crystals: Molding the Flow of Light (Princeton University Press, Princeton, NJ, 2011).

[17] T. K. Paraïso, M. Kalaee, L. Zang, H. Pfeifer, F. Marquardt, and O. Painter, Phys. Rev. X 5, 041024 (2015).

[18] M. Ludwig, A. H. Safavi-Naeini, O. Painter, and F. Marquardt, Phys. Rev. Lett. 109, 063601 (2012).

[19] See Supplemental Material http://link.aps.org/supplemental/ 10.1103/PhysRevLett.118.133603 for the derivation of Eqs. (3)-(4) and further details on the validity of the approximations involved, on the role of the other supermodes in the three-cavity system, on the generation of the nonclassical states and on the numerical simulations of the structure in Fig. 2(g)-2(h), which includes Refs. [20-25].

[20] J. C. Lee, D. O. Bracher, S. Cui, K. Ohno, C. A. McLellan, X. Zhang, P. Andrich, B. Alemán, K. J. Russell, A. P. Magyar, I. Aharonovich, A. B. Jayich, D. Awschalom, and E. L. Hu, Appl. Phys. Lett. 105, 261101 (2014).

[21] M. A. Nielsen and I. L. Chuang, Quantum Computation and Quantum Information (Cambridge University Press, Cambridge, 2000), Vol. 2, pp. 23.

[22] A. Faraon, C. Santori, Z. Huang, V. M. Acosta, and R. G. Beausoleil, Phys. Rev. Lett. 109, 033604 (2012).

[23] P. Lodahl, S. Mahmoodian, and S. Stobbe, Rev. Mod. Phys. 87, 347 (2015)

[24] L. Robledo, H. Bernien, I. van Weperen, and R. Hanson, Phys. Rev. Lett. 105, 177403 (2010).

[25] C. K. Law and J. H. Eberly, Phys. Rev. Lett. 76, 1055 (1996).

[26] R. Johne, R. Schutjens, S. Fattah Poor, C.-Y. Jin, and A. Fiore, Phys. Rev. A 91, 063807 (2015).

[27] N. Caselli, F. Riboli, F. La China, A. Gerardino, L. Li, E. H. Linfield, F. Pagliano, A. Fiore, F. Intonti, and M. Gurioli, ACS Photonics 2, 565 (2015).

[28] J. Chan, T. M. Alegre, A. H. Safavi-Naeini, J. T. Hill, A. Krause, S. Groblacher, M. Aspelmeyer, and O. Painter, Nature (London) 478, 89 (2011).

[29] M. J. Burek, J. D. Cohen, S. M. Meenehan, T. Ruelle, S. Meesala, J. Rochman, H. A. Atikian, M. Markham, D. J. Twitchen, M. D. Lukin, O. Painter, and M. Lončar, Optica 3, 1404 (2016).

[30] J. Johansson, P. Nation, and F. Nori, Comput. Phys. Commun. 183, 1760 (2012).

[31] A. Goban, C.-L. Hung, S.-P. Yu, J. Hood, J. Muniz, J. Lee, M. Martin, A. McClung, K. Choi, D. Chang, O. Painter, and H. Kimble, Nat. Commun. 5, 3808 (2014).

[32] J. Thompson, T. Tiecke, N. de Leon, J. Feist, A. Akimov, M. Gullans, A. Zibrov, V. Vuletić, and M. Lukin, Science 340, 1202 (2013).

[33] K. Hammerer, M. Wallquist, C. Genes, M. Ludwig, F. Marquardt, P. Treutlein, P. Zoller, J. Ye, and H. J. Kimble, Phys. Rev. Lett. 103, 063005 (2009). 


\title{
Supplemental information for Coherent Atom-Phonon Interaction through Mode Field Coupling in Hybrid Optomechanical Systems
}

\author{
Michele Cotrufo* and Andrea Fiore \\ Department of Applied Physics, Eindhoven University of Technology, \\ 5600 MB Eindhoven, The Netherlands \\ Ewold Verhagen \\ Center for Nanophotonics, AMOLF, \\ Science Park 104, 1098 XG Amsterdam, The Netherlands
}

* Electronic address: m.cotrufo@tue.nl 


\section{CONTENTS}

I. Density matrix calculations, numerical setup 2

II. Derivation of the Mode Field Coupling for the two-cavity system 3

III. Derivation of the Mode Field Coupling for the three-cavity system 5

IV. Validity of the linear approximation in the derivation of the Mode Field Coupling 8

V. Emitter decay rate in the three-cavity system 9

VI. Additional radiative losses introduced by the supermodes $\hat{a}_{+}$and $\hat{a}_{-} \quad 10$

VII. Selective pumping of the optical mode of interest in the three-cavity system 10

VIII. Three-cavity system Hamiltonian at large driving 11

IX. Influence of cavity population and dephasing on the fidelity of the one-phonon $\begin{array}{ll}\text { state creation } & 11\end{array}$

X. Creation of other nonclassical mechanical states 12

X.1. One-cavity model with MFC 12

$\begin{array}{ll}\text { X.2. Three-cavity model } & 14\end{array}$

$\begin{array}{ll}\text { XI. Role of the other two mechanical supermodes } & 15\end{array}$

$\begin{array}{ll}\text { XII. Design of the proposed structure } & 17\end{array}$

$\begin{array}{ll}\text { XIII. Tripartite interaction in a Fabry-Perot cavity } & 18\end{array}$

$\begin{array}{ll}\text { References } & 19\end{array}$

\section{DENSITY MATRIX CALCULATIONS, NUMERICAL SETUP}

The non-Hermitian evolution of the different systems considered in this work has been calculated with a Master equation approach, in which the different dissipative channels are described by proper Lindblad terms. For a given Hamiltonian $\hat{H}$, we calculate the temporal evolution of the density matrix $\rho$ through the equation

$$
\begin{aligned}
\dot{\hat{\rho}} & =-\frac{i}{\hbar}[\hat{H}, \hat{\rho}]+\sum_{i} \frac{\kappa_{i}}{2}\left(2 \hat{a}_{i} \hat{\rho} \hat{a}_{i}^{\dagger}-\left\{\hat{a}_{i}^{\dagger} \hat{a}_{i}, \hat{\rho}\right\}\right)+\frac{\Gamma_{\mathrm{M}}\left(n_{\mathrm{th}}+1\right)}{2}\left(2 \hat{b} \hat{\rho} \hat{b}^{\dagger}-\left\{\hat{b}^{\dagger} \hat{b}, \hat{\rho}\right\}\right)+ \\
& +\frac{\Gamma_{\mathrm{M}} n_{\mathrm{th}}}{2}\left(2 \hat{b}^{\dagger} \hat{\rho} \hat{b}-\left\{\hat{b} \hat{b}^{\dagger}, \hat{\rho}\right\}\right)+\frac{\Gamma}{2}\left(2 \hat{\sigma}_{-} \hat{\rho} \hat{\sigma}_{+}-\left\{\hat{\sigma}_{+} \sigma_{-}, \hat{\rho}\right\}\right)+\frac{\Gamma^{*}}{2}\left(\hat{\sigma}_{z} \hat{\rho} \hat{\sigma}_{z}-\hat{\rho}\right)
\end{aligned}
$$

where the sum refers to all the optical cavities considered, $\kappa_{i}$ is decay rate of the $\mathrm{i}$-th cavity, $\Gamma_{\mathrm{M}}$ is the decay rate of the mechanical mode, $n_{\mathrm{th}}$ is the average phonon number of the external bath, and $\Gamma$ and $\Gamma^{*}$ are the decay rate and pure dephasing rate of the emitter, respectively. The brackets [,] and $\{$,$\} indicate commutation and anti-commutation$ of the operators, respectively. The master equation has been solved numerically with the opensource Python framework QuTIP [1]. For the numerical calculations, the dimensions 
of the Fock spaces of the optical cavities and mechanical resonator need to be truncated. For the calculations of the emitter-phonon swapping, the Fock spaces of the mechanical oscillator and optical cavities all have dimensions of 2 . We verified that no appreciable numerical differences arise for larger Fock spaces dimensions. For the cooling calculations, the Fock space of the mechanical resonator has a dimension of 15, to ensure that a thermal state with $n_{\text {th }}=4$ can be properly described.

Both in the in main text and in this SI we frequently use the concept of fidelity to quantify the closeness of a created state and a target one. The fidelity is defined as $\mathrm{F}\left(\hat{\rho}_{A}, \hat{\rho}_{B}\right) \equiv$ $\operatorname{tr}\left(\sqrt{\left(\hat{\rho}_{A}\right)^{1 / 2} \hat{\rho}_{B}\left(\hat{\rho}_{A}\right)^{1 / 2}}\right)[2]$, where $\hat{\rho}_{A}$ and $\hat{\rho}_{B}$ are the density matrices of the states to be compared and "tr" denotes the trace.

\section{DERIVATION OF THE MODE FIELD COUPLING FOR THE TWO-CAVITY SYSTEM}

The general model describing mode field coupling in a two-cavity system is schematically depicted in fig. S1. We consider two identical optomechanical systems, denoted left (L) and right $(\mathrm{R})$, each composed of an optical cavity with frequency $\omega_{\mathrm{c}}$ and a mechanical resonator with frequency $\Omega_{\mathrm{M}}$. In each system, the cavity and the resonator are dispersively coupled at a rate $g_{0}$. We describe the fields in the two optical cavities with the annihilation operators $\hat{a}_{\mathrm{L}}$ and $\hat{a}_{\mathrm{R}}$, and the two mechanical resonators with annihilation operators $\hat{b}_{\mathrm{L}}$ and $\hat{b}_{\mathrm{R}}$. The two optical cavities are coupled with a rate $J$, while the two mechanical resonators are coupled with a rate $J_{\mathrm{M}}$. Finally, a two-level emitter is placed in the left cavity, and interacts with the field $\hat{a}_{\mathrm{L}}$ with a coupling rate $g$.

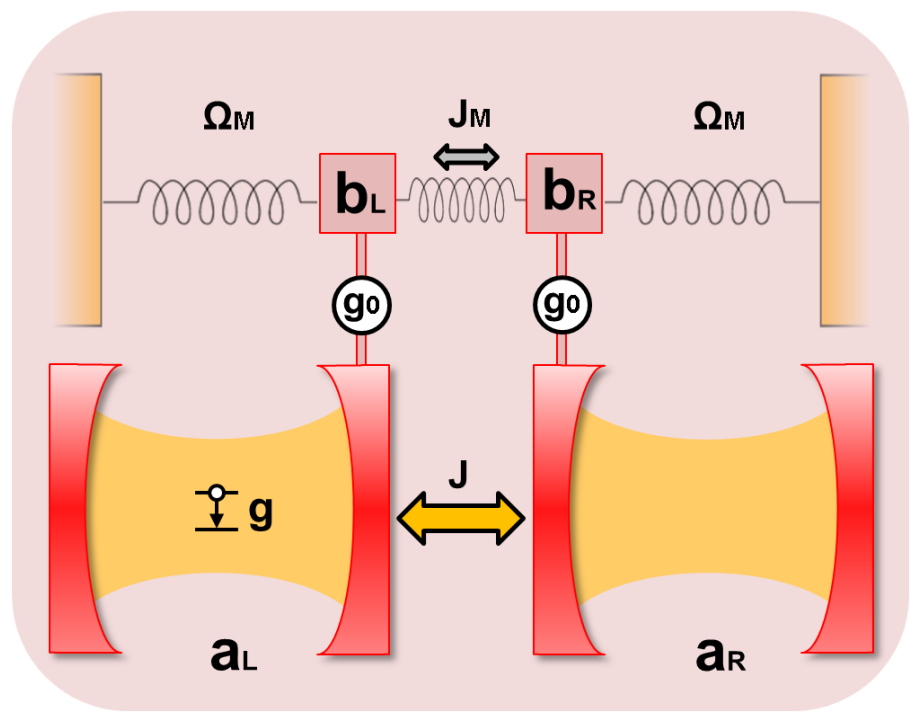

FIG. S1. Schematic of the two-cavity system. Two identical optical cavities (each denoted by a couple of red mirrors) interact with each other at a rate $J$. Each cavity is dispersively coupled to a separate mechanical resonator with an optomechanical coupling rate $g_{0}$. The resonators are identical and have frequency $\Omega_{\mathrm{M}}$. The two mechanical resonators are additionally coupled to each other at a rate $J_{\mathrm{M}}$. An emitter is placed in the left optical cavity and interacts with one of its optical modes with a coupling rate $g$. 
The full Hamiltonian reads

$$
\begin{aligned}
\hat{H}= & {\left[\omega_{\mathrm{c}}-g_{0}\left(\hat{b}_{\mathrm{L}}^{\dagger}+\hat{b}_{\mathrm{L}}\right)\right] \hat{a}_{\mathrm{L}}^{\dagger} \hat{a}_{\mathrm{L}}+\left[\omega_{\mathrm{c}}-g_{0}\left(\hat{b}_{\mathrm{R}}^{\dagger}+\hat{b}_{\mathrm{R}}\right)\right] \hat{a}_{\mathrm{R}}^{\dagger} \hat{a}_{\mathrm{R}}+\Omega_{\mathrm{M}}\left(\hat{b}_{\mathrm{L}}^{\dagger} \hat{b}_{\mathrm{L}}+\hat{b}_{\mathrm{R}}^{\dagger} \hat{b}_{\mathrm{R}}\right)+\frac{\omega_{\mathrm{A}}}{2} \hat{\sigma}_{z}+} \\
& +J\left(\hat{a}_{R}^{\dagger} \hat{a}_{\mathrm{L}}+\text { h.c. }\right)+J_{\mathrm{M}}\left(\hat{b}_{R}^{\dagger} \hat{b}_{\mathrm{L}}+\text { h.c. }\right)+g\left(\hat{\sigma}_{+} \hat{a}_{\mathrm{L}}+\text { h.c. }\right) .
\end{aligned}
$$

We now introduce mechanical supermodes $\hat{b}_{ \pm}=\frac{1}{\sqrt{2}}\left(\hat{b}_{\mathrm{L}} \pm \hat{b}_{\mathrm{R}}\right)$. Substituting these in the Hamiltonian, we obtain

$$
\begin{aligned}
\hat{H}= & {\left[\omega_{\mathrm{c}}-\frac{g_{0}}{\sqrt{2}}\left(\hat{x}_{+}+\hat{x}_{-}\right)\right] \hat{a}_{\mathrm{L}}^{\dagger} \hat{a}_{\mathrm{L}}+\left[\omega_{\mathrm{c}}-\frac{g_{0}}{\sqrt{2}}\left(\hat{x}_{+}-\hat{x}_{-}\right)\right] \hat{a}_{\mathrm{R}}^{\dagger} \hat{a}_{\mathrm{R}}+} \\
& +\left(\Omega_{\mathrm{M}}+J_{\mathrm{M}}\right) \hat{b}_{+}^{\dagger} \hat{b}_{+}+\left(\Omega_{\mathrm{M}}-J_{\mathrm{M}}\right) \hat{b}_{-}^{\dagger} \hat{b}_{-}+\frac{\omega_{\mathrm{A}}}{2} \hat{\sigma}_{z}+ \\
& +J\left(\hat{a}_{R}^{\dagger} \hat{a}_{\mathrm{L}}+\text { h.c. }\right)+g\left(\hat{\sigma}_{+} \hat{a}_{\mathrm{L}}+\text { h.c. }\right)
\end{aligned}
$$

where we have defined the dimensionless position operators of the mechanical supermodes $\hat{x}_{ \pm}=\hat{b}_{ \pm}^{\dagger}+\hat{b}_{ \pm}$. The mechanical mode $\hat{b}_{-}$interacts dispersively with the modes $\hat{a}_{\mathrm{R}}$ and $\hat{a}_{\mathrm{L}}$ with equal and opposite coupling rate $\pm g_{0} / \sqrt{2}$. In the following, we will neglect the presence of the other mechanical supermode, $\hat{b}_{+}$. For simplicity, we replace $\hat{b}_{-} \rightarrow \hat{b}$ and $\left(\Omega_{\mathrm{M}}-J_{\mathrm{M}}\right) \rightarrow \Omega_{\mathrm{M}}$. We moreover define the operator $\hat{\Delta}=\frac{g_{0}}{\sqrt{2}}\left(\hat{b}^{\dagger}+\hat{b}\right)$. The new Hamiltonian reads

$$
\begin{aligned}
\hat{H}= & -\hat{\Delta} \hat{a}_{\mathrm{L}}^{\dagger} \hat{a}_{\mathrm{L}}+\hat{\Delta} \hat{a}_{\mathrm{R}}^{\dagger} \hat{a}_{\mathrm{R}}+\Omega_{\mathrm{M}} \hat{b}^{\dagger} \hat{b}+\frac{\omega_{\mathrm{A}}-\omega_{\mathrm{c}}}{2} \hat{\sigma}_{z}+ \\
& +J\left(\hat{a}_{R}^{\dagger} \hat{a}_{\mathrm{L}}+\text { h.c. }\right)+g\left(\hat{\sigma}_{+} \hat{a}_{\mathrm{L}}+\text { h.c. }\right)
\end{aligned}
$$

where we also performed a unitary transformation $\hat{H} \rightarrow \hat{U}(t) \hat{H} \hat{U}^{\dagger}(t)-i \hat{U}(t) \frac{\partial \hat{U}(t)^{\dagger}}{\partial t}$, with $\hat{U}(t)=\exp \left[-i \omega_{\mathrm{c}} t\left(\hat{a}_{\mathrm{L}}^{\dagger} \hat{a}_{\mathrm{L}}++\hat{a}_{\mathrm{R}}^{\dagger} \hat{a}_{\mathrm{R}}+\hat{\sigma}_{+} \hat{\sigma}_{-}\right)\right]$. Assuming a quasi-static approximation for $\hat{\Delta}$, valid in the limit $J \gg \Omega_{\mathrm{M}}$, we can diagonalize the optical part of the Hamiltonian [3]. The optical supermodes are defined by

$$
\hat{a}_{+}=\alpha \hat{a}_{\mathrm{L}}+\beta \hat{a}_{\mathrm{R}}, \hat{a}_{-}=\beta \hat{a}_{\mathrm{L}}-\alpha \hat{a}_{\mathrm{R}}
$$

where $\alpha$ and $\beta$ are operators defined by

$$
\alpha=\frac{\sqrt{\hat{\Delta}^{2}+J^{2}}+\hat{\Delta}}{\sqrt{\left(\sqrt{\hat{\Delta}^{2}+J^{2}}+\hat{\Delta}\right)^{2}+J^{2}}}, \quad \beta=\frac{J}{\sqrt{\left(\sqrt{\hat{\Delta}^{2}+J^{2}}+\hat{\Delta}\right)^{2}+J^{2}}} .
$$

In the supermode basis, the Hamiltonian is

$$
\begin{aligned}
\hat{H}= & \sqrt{J^{2}+\hat{\Delta}^{2}} \hat{a}_{+}^{\dagger} \hat{a}_{+}-\sqrt{J^{2}+\hat{\Delta}^{2}} \hat{a}_{-}^{\dagger} \hat{a}_{-}+\frac{\omega_{\mathrm{A}}-\omega_{\mathrm{c}}}{2} \hat{\sigma}_{z}+\Omega_{\mathrm{M}} \hat{b}^{\dagger} \hat{b}+ \\
& +g \alpha\left(\hat{\sigma}_{+} \hat{a}_{+}+\text {h.c. }\right)+g \beta\left(\hat{\sigma}_{+} \hat{a}_{-}+\text {h.c. }\right) .
\end{aligned}
$$


Up to the first order in $\hat{\Delta} / J$, the coefficients $\alpha$ and $\beta$ read

$$
\alpha=\frac{1}{\sqrt{2}}\left(1+\frac{\hat{\Delta}}{2 J}\right)+\mathcal{O}\left(\frac{\hat{\Delta}}{J}\right)^{2}, \quad \beta=\frac{1}{\sqrt{2}}\left(1-\frac{\hat{\Delta}}{2 J}\right)+\mathcal{O}\left(\frac{\hat{\Delta}}{J}\right)^{2} .
$$

By inserting these expansions in the Hamiltonian in eq. S7, and expressing again $\hat{\Delta}$ as a function of $\hat{b}$ and $\hat{b}^{\dagger}$, we get

$$
\begin{aligned}
\hat{H}= & \sqrt{J^{2}+\hat{\Delta}^{2}} \hat{a}_{+}^{\dagger} \hat{a}_{+}-\sqrt{J^{2}+\hat{\Delta}^{2}} \hat{a}_{-}^{\dagger} \hat{a}_{-}+\frac{\omega_{\mathrm{A}}-\omega_{\mathrm{c}}}{2} \hat{\sigma}_{z}+\Omega_{\mathrm{M}} \hat{b}^{\dagger} \hat{b}+ \\
& +\frac{g}{\sqrt{2}}\left(\hat{\sigma}_{+} \hat{a}_{+}+\text {h.c. }\right)+\frac{g}{\sqrt{2}}\left(\hat{\sigma}_{+} \hat{a}_{-}+\text {h.c. }\right)+ \\
& +\frac{g g_{0}}{4 J}\left(\hat{b}^{\dagger}+\hat{b}\right)\left(\hat{\sigma}_{+} \hat{a}_{+}+\text {h.c. }\right)-\frac{g g_{0}}{4 J}\left(\hat{b}^{\dagger}+\hat{b}\right)\left(\hat{\sigma}_{+} \hat{a}_{-}+\text {h.c. }\right) .
\end{aligned}
$$

The second row describes the Rabi interaction of the emitter with the two optical supermodes, with a coupling rate $g / \sqrt{2}$. As mentioned in the main text, in the two-cavity system this interaction is unavoidable and is due to the fact that both optical supermodes have nonzero field in both cavities for all finite detunings. The third row describes the tripartite interaction between the emitter, the phonon and the optical supermodes.

\section{DERIVATION OF THE MODE FIELD COUPLING FOR THE THREE-CAVITY SYSTEM}

The model for the three-cavity case is schematically depicted in fig. S2. The three identical optical cavities, denoted left $(\mathrm{L})$, target $(\mathrm{T})$ and right $(\mathrm{R})$ are dispersively coupled at a rate $g_{0}$ with a separate mechanical resonator, in similar fasion as in the previous section. The three mechanical resonators are assumed identical and with frequency $\Omega_{\mathrm{M}}$. We describe the three optical cavities with the annihilation operators $\hat{a}_{\mathrm{L}}, \hat{a}_{\mathrm{T}}$ and $\hat{a}_{\mathrm{R}}$, and the three resonators with annihilation operators $\hat{b}_{\mathrm{L}}, \hat{b}_{\mathrm{T}}$ and $\hat{b}_{\mathrm{R}}$. The optical cavity $\mathrm{T}$ is coupled to the cavities $\mathrm{L}$ and $\mathrm{R}$ with a rate $J$, while the mechanical resonator $\hat{b}_{\mathrm{T}}$ is coupled with a rate $J_{\mathrm{M}}$ to the resonators $\hat{b}_{\mathrm{R}}$ and $\hat{b}_{\mathrm{L}}$. Finally, a two-level emitter is placed in the target cavity, and interacts with the field $\hat{a}_{\mathrm{T}}$ with a coupling rate $g$.

The full Hamiltonian reads

$$
\begin{aligned}
\hat{H}= & {\left[\omega_{\mathrm{c}}-g_{0}\left(\hat{b}_{\mathrm{L}}^{\dagger}+\hat{b}_{\mathrm{L}}\right)\right] \hat{a}_{\mathrm{L}}^{\dagger} \hat{a}_{\mathrm{L}}+\left[\omega_{\mathrm{c}}-g_{0}\left(\hat{b}_{\mathrm{T}}^{\dagger}+\hat{b}_{\mathrm{T}}\right)\right] \hat{a}_{\mathrm{T}}^{\dagger} \hat{a}_{\mathrm{T}}+\left[\omega_{\mathrm{c}}-g_{0}\left(\hat{b}_{\mathrm{R}}^{\dagger}+\hat{b}_{\mathrm{R}}\right)\right] \hat{a}_{\mathrm{R}}^{\dagger} \hat{a}_{\mathrm{R}}+} \\
& +\Omega_{\mathrm{M}}\left(\hat{b}_{\mathrm{L}}^{\dagger} \hat{b}_{\mathrm{L}}+\hat{b}_{\mathrm{T}}^{\dagger} \hat{b}_{\mathrm{T}}+\hat{b}_{\mathrm{R}}^{\dagger} \hat{b}_{\mathrm{R}}\right)+\frac{\omega_{\mathrm{A}}}{2} \hat{\sigma}_{z}+ \\
& +J\left[\hat{a}_{\mathrm{T}}^{\dagger}\left(\hat{a}_{\mathrm{R}}+\hat{a}_{\mathrm{L}}\right)+\text { h.c. }\right]+J_{\mathrm{M}}\left[\hat{b}_{\mathrm{T}}^{\dagger}\left(\hat{b}_{\mathrm{R}}+\hat{b}_{\mathrm{L}}\right)+\text { h.c. }\right]+g\left(\hat{\sigma}_{+} \hat{a}_{\mathrm{T}}+\text { h.c. }\right) .
\end{aligned}
$$

The mechanical part of this Hamiltonian can be diagonalized by introducing the modes

$$
\begin{aligned}
& \hat{b}_{0}=\frac{1}{\sqrt{2}}\left(\hat{b}_{\mathrm{L}}-\hat{b}_{\mathrm{R}}\right), \\
& \hat{b}_{+}=\frac{1}{\sqrt{2}} \hat{b}_{\mathrm{T}}+\frac{1}{2}\left(\hat{b}_{\mathrm{R}}+\hat{b}_{\mathrm{L}}\right), \\
& \hat{b}_{-}=-\frac{1}{\sqrt{2}} \hat{b}_{\mathrm{T}}+\frac{1}{2}\left(\hat{b}_{\mathrm{R}}+\hat{b}_{\mathrm{L}}\right) .
\end{aligned}
$$




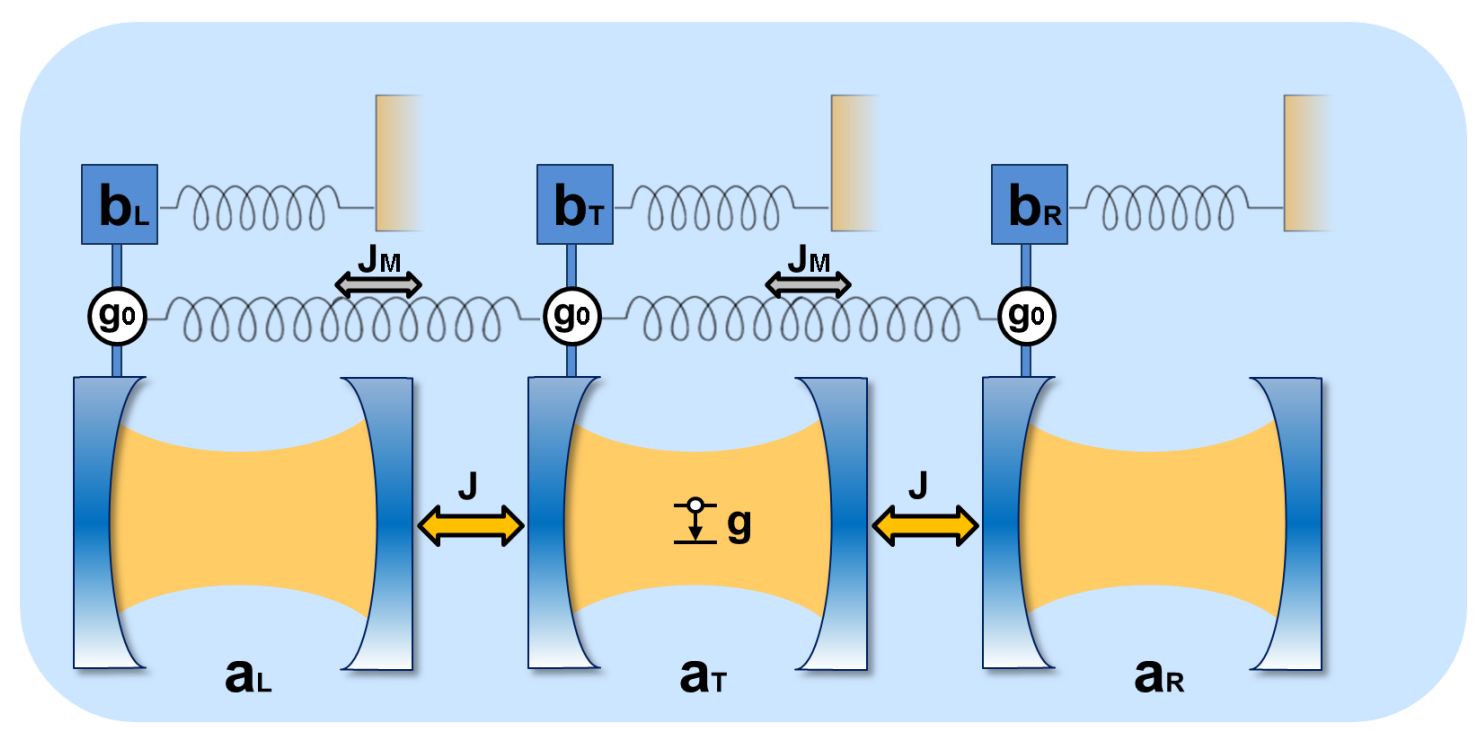

FIG. S2. Schematic of the three-cavity system. Three identical optical cavities (each denoted by a couple of blue mirrors) are arranged such that the central one interacts with the two lateral ones with a rate $J$. Each cavity is dispersively coupled to a separate resonator with an optomechanical coupling rate $g_{0}$. The resonators are identical and have frequency $\Omega_{\mathrm{M}}$. The central mechanical resonator is additionally coupled to the other two mechanical resonators with a rate $J_{\mathrm{M}}$. An emitter is placed in the central optical cavity and interacts with its optical mode with a coupling rate $g$.

The transformed Hamiltonian reads

$$
\begin{aligned}
\hat{H}= & \omega_{\mathrm{c}}\left(\hat{a}_{\mathrm{L}}^{\dagger} \hat{a}_{\mathrm{L}}+\hat{a}_{\mathrm{T}}^{\dagger} \hat{a}_{\mathrm{T}}+\hat{a}_{\mathrm{R}}^{\dagger} \hat{a}_{\mathrm{R}}\right)+\Omega_{\mathrm{M}} \hat{b}_{0}^{\dagger} \hat{b}_{0}+\left(\Omega_{\mathrm{M}}+\sqrt{2} J_{\mathrm{M}}\right) \hat{b}_{+}^{\dagger} \hat{b}_{+}+\left(\Omega_{\mathrm{M}}-\sqrt{2} J_{\mathrm{M}}\right) \hat{b}_{-}^{\dagger} \hat{b}_{-}+\frac{\omega_{\mathrm{A}}}{2} \hat{\sigma}_{z}+ \\
& +\frac{g_{0}}{\sqrt{2}} \hat{x}_{0}\left(\hat{a}_{\mathrm{R}}^{\dagger} \hat{a}_{\mathrm{R}}-\hat{a}_{\mathrm{L}}^{\dagger} \hat{a}_{\mathrm{L}}\right)+\frac{g_{0}}{2}\left(\hat{x}_{+}-\hat{x}_{-}\right)\left(\hat{a}_{\mathrm{L}}^{\dagger} \hat{a}_{\mathrm{L}}+\hat{a}_{\mathrm{R}}^{\dagger} \hat{a}_{\mathrm{R}}\right)-\frac{g_{0}}{2}\left(\hat{x}_{+}-\hat{x}_{-}\right) \hat{a}_{\mathrm{T}}^{\dagger} \hat{a}_{\mathrm{T}}+ \\
& +J\left[\hat{a}_{\mathrm{T}}^{\dagger}\left(\hat{a}_{\mathrm{R}}+\hat{a}_{\mathrm{L}}\right)+\text { h.c. }\right]+g\left(\hat{\sigma}_{+} \hat{a}_{\mathrm{T}}+\text { h.c. }\right),
\end{aligned}
$$

where we have defined the dimensionless position operators of the mechanical supermodes $\hat{x}_{ \pm}=\hat{b}_{ \pm}^{\dagger}+\hat{b}_{ \pm}$and $\hat{x}_{0}=\hat{b}_{0}^{\dagger}+\hat{b}_{0}$. We now focus only on the mechanical mode $\hat{b}_{0}$ with the assumption that, under the resonant condition $\omega_{\mathrm{A}}=\omega_{\mathrm{c}}+\Omega_{\mathrm{M}}$, the terms involving the other mechanical modes are negligible (this assumption is numerically verified in section $\mathrm{XI})$. For simplicity, we redefine $\hat{b}_{0} \rightarrow \hat{b}$. Moreover, we perform a unitary transformation $\hat{H} \rightarrow \hat{U}(t) \hat{H} \hat{U}^{\dagger}(t)-i \hat{U}(t) \frac{\partial \hat{U}(t)^{\dagger}}{\partial t}$, with $\hat{U}(t)=\exp \left[-i \omega_{\mathrm{c}} t\left(\hat{a}_{\mathrm{L}}^{\dagger} \hat{a}_{\mathrm{L}}+\hat{a}_{\mathrm{T}}^{\dagger} \hat{a}_{\mathrm{T}}+\hat{a}_{\mathrm{R}}^{\dagger} \hat{a}_{\mathrm{R}}+\hat{\sigma}_{+} \hat{\sigma}_{-}\right)\right]$. The new Hamiltonian reads

$$
\begin{aligned}
\hat{H}= & -\hat{\Delta} \hat{a}_{\mathrm{L}}^{\dagger} \hat{a}_{\mathrm{L}}+\hat{\Delta} \hat{a}_{\mathrm{R}}^{\dagger} \hat{a}_{\mathrm{R}}+\Omega_{\mathrm{M}} \hat{b}^{\dagger} \hat{b}++\frac{\omega_{\mathrm{A}}-\omega_{\mathrm{c}}}{2} \hat{\sigma}_{z}+ \\
& +J\left[\hat{a}_{\mathrm{T}}^{\dagger}\left(\hat{a}_{\mathrm{R}}+\hat{a}_{\mathrm{L}}\right)+\text { h.c. }\right]+g\left(\hat{\sigma}_{+} \hat{a}_{\mathrm{T}}+\text { h.c. }\right)
\end{aligned}
$$

where we defined $\hat{\Delta}=g_{0} \hat{x}_{0} / \sqrt{2}$. We note that the selected mechanical mode has equal and opposite dispersive coupling with the optical cavities $\mathrm{L}$ and $\mathrm{R}$, at a rate $\pm g_{0} / \sqrt{2}$, while it does not affect the optical cavity T. We again assume a quasi-static approximation for $\hat{\Delta}$, valid in the limit $J \gg \Omega_{\mathrm{M}}$, to diagonalize the optical part of the Hamiltonian [3]. The three 
optical supermodes are defined in this case by

$$
\begin{aligned}
& \hat{a}_{0}=-\epsilon \hat{a}_{\mathrm{L}}+\beta \hat{a}_{\mathrm{T}}+\epsilon \hat{a}_{\mathrm{R}}, \\
& \hat{a}_{+}=\eta \hat{a}_{\mathrm{L}}-\epsilon \hat{a}_{\mathrm{T}}+\mu \hat{a}_{\mathrm{R}}, \\
& \hat{a}_{-}=\mu \hat{a}_{\mathrm{L}}+\epsilon \hat{a}_{\mathrm{T}}+\eta \hat{a}_{\mathrm{R}},
\end{aligned}
$$

where $\epsilon, \beta, \mu$ and $\eta$ are operators. In particular,

$$
\epsilon=\frac{1}{\sqrt{2+(\hat{\Delta} / J)^{2}}}, \quad \beta=\frac{\hat{\Delta} / J}{\sqrt{2+(\hat{\Delta} / J)^{2}}} .
$$

The functions $\eta$ and $\mu$ satisfy $\eta(\hat{\Delta}, J)=\mu(-\hat{\Delta}, J)$. Their expressions are more complicated and not reported here, as they are not needed in the following. The Hamiltonian in the supermode basis reads

$$
\begin{aligned}
\hat{H}= & \sqrt{2 J^{2}+\hat{\Delta}^{2}}\left(\hat{a}_{+}^{\dagger} \hat{a}_{+}-\hat{a}_{-}^{\dagger} \hat{a}_{-}\right)+\Omega_{\mathrm{M}} \hat{b}^{\dagger} \hat{b}++\frac{\omega_{\mathrm{A}}-\omega_{\mathrm{c}}}{2} \hat{\sigma}_{z}+ \\
& +g \beta\left(\hat{\sigma}_{+} \hat{a}_{0}+\text { h.c. }\right)+g \epsilon\left[\hat{\sigma}_{+}\left(\hat{a}_{+}-\hat{a}_{-}\right)+\text {h.c. }\right] .
\end{aligned}
$$

Up to the first order in $\hat{\Delta} / J$, the functions $\beta$ and $\epsilon$ are

$$
\beta=\frac{1}{\sqrt{2}} \frac{\hat{\Delta}}{J}+\mathcal{O}\left(\frac{\hat{\Delta}}{J}\right)^{3}, \quad \epsilon=\frac{1}{\sqrt{2}}+\mathcal{O}\left(\frac{\hat{\Delta}}{J}\right)^{2} .
$$

By inserting these expansions in the Hamiltonian in eq. S16, and expressing again $\hat{\Delta}$ as a function of $\hat{b}$ and $\hat{b}^{\dagger}$, we get

$$
\begin{aligned}
\hat{H}= & \sqrt{2 J^{2}+\hat{\Delta}^{2}}\left(\hat{a}_{+}^{\dagger} \hat{a}_{+}-\hat{a}_{-}^{\dagger} \hat{a}_{-}\right)+\Omega_{\mathrm{M}} \hat{b}^{\dagger} \hat{b}++\frac{\omega_{\mathrm{A}}-\omega_{\mathrm{c}}}{2} \hat{\sigma}_{z}+ \\
& +\frac{g g_{0}}{2 J}\left(\hat{b}^{\dagger}+\hat{b}\right)\left(\hat{\sigma}_{+} \hat{a}_{0}+\text { h.c. }\right)+\frac{g}{\sqrt{2}}\left[\hat{\sigma}_{+}\left(\hat{a}_{+}-\hat{a}_{-}\right)+\text {h.c. }\right] .
\end{aligned}
$$

The first term of the second row describes the mode field coupling between the emitter, the phonon and the mode $\hat{a}_{0}$, with a coupling rate $\gamma=\frac{g g_{0}}{2 J}$. The second term of the second row describes a Rabi interaction between the emitter and the other two optical supermodes. As confirmed by the numerical simulations based on the full Hamiltonian in eq. S13 (see main text), the effect of these terms is negligible when the spectral separation between the supermodes is much larger than the mechanical frequency $\left(\sqrt{2} J \gg \Omega_{\mathrm{M}}\right)$. However, for finite optical linewidths $(\kappa)$, the presence of the modes $\hat{a}_{ \pm}$will introduce additional decay channels for the QE, as explained below.

To verify the validity of the effective Hamiltonian derived in eq. S18, and in particular the presence of the predicted tripartite QE-phonon-photon interaction, we numerically calculated the evolution of the system dictated by the Hamiltonian in eq. S13, i.e., without introducing the optical supermodes. We start with the QE excited, we use the parameters mentioned in the main text, $\left\{\omega_{\mathrm{c}}, \Omega_{\mathrm{M}}, g, g_{0}, J\right\}=2 \pi \cdot\left\{4.7 \cdot 10^{5}, 14,20,0.004,360\right\} \mathrm{GHz}$, and we set the resonant condition $\omega_{\mathrm{A}}=\omega_{\mathrm{c}}+\Omega_{\mathrm{M}}$. As shown in fig. S3a, the lossless evolution of the system agrees perfectly with that of the MFC Hamiltonian (fig. 1c of the main text): the system oscillates between the state in which only the QE is excited and the state in which 
the QE is in the ground state and one photon and one phonon are present. The oscillation period verifies the predicted value of $T=\pi / \gamma=2 \pi J /\left(g g_{0}\right)=4.5 \mu \mathrm{s}$. This confirms that, for realistic choices of parameters, a purely tripartite interaction is obtained in the three-cavity system, as predicted by the effective Hamiltonian in eq. S18.

Even in presence of losses, nonclassical states can be obtained. In the main text we have shown how the losses can be overcome by strongly pumping the mode $\hat{a}_{0}$ (fig. 3 a of the main text), and the one-phonon state can be created with high fidelity, $\mathrm{F}=0.86$. The Wigner map of the mechanical state created in fig. 3a is shown in fig. S3b. The nonclassical nature of the state is evident from the negativity of the Wigner map.
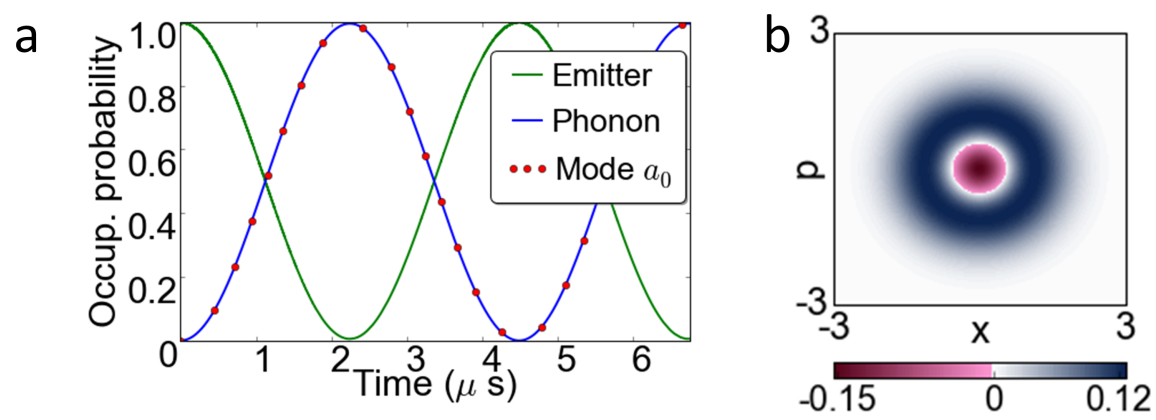

FIG. S3. (a) Lossless evolution dictated by the Hamiltonian in eq. S13, starting from an excited $\mathrm{QE}$ and with a red-detuned cavity $\left(\omega_{\mathrm{A}}=\omega_{\mathrm{c}}+\Omega_{\mathrm{M}}\right)$. The parameters are $\left\{\omega_{\mathrm{c}}, \Omega_{\mathrm{M}}, g, g_{0}, J\right\}=$ $2 \pi \cdot\left\{4.7 \cdot 10^{5}, 14,20,0.004,360\right\} \mathrm{GHz}$. (b) Wigner map of the mechanical state created in fig. 3a of the main paper.

\section{VALIDITY OF THE LINEAR APPROXIMATION IN THE DERIVATION OF THE MODE FIELD COUPLING}

In deriving the Hamiltonian of both the two-cavity and three-cavity systems we neglected terms of second or higher order in $\hat{\Delta} / J$ (eqs. S8 and S17). This approximation sets, in principle, a limit on the mechanical nonclassical state which can be created via the MFC, because for a generic state $|\Psi\rangle$ the expectation value of the operator $\hat{\Delta}$ may be not negligible with respect to $J$. However, as we show here, this limit is extremely large. We can consider a generic state of the mechanical resonator, $|\Psi\rangle=\sum_{n=0}^{\infty} C_{n}|n\rangle$, where the $C_{n}$ are arbitrary coefficients and $|n\rangle$ are the Fock states. Since the expectation value of the operator $\hat{\Delta}=g_{0} / \sqrt{2}\left(\hat{b}+\hat{b}^{\dagger}\right)$ is zero in any Fock state, we instead consider the quantity $\sqrt{\left\langle\hat{\Delta}^{2}\right\rangle}$. Requiring that $\sqrt{\left\langle\hat{\Delta}^{2}\right\rangle} \ll J$ leads to

$$
\sum_{n=0}^{\infty}\left[\left(C_{n+2}^{*} C_{n}+C_{n+2} C_{n}^{*}\right) \sqrt{(n+1)(n+2)}+\left|C_{n}\right|^{2}(2 n+1)\right] \ll \frac{2 J^{2}}{g_{0}^{2}} .
$$

For a Fock state (i.e., $C_{n}=1, C_{m \neq n}=0$ ), this inequality leads to $n \ll\left(J / g_{0}\right)^{2}$. With the parameters used in the main text, this translates into $n \ll 10^{10}$, which ensures us that the MFC allows generating nonclassical mechanical states in extremely large Fock spaces. Essentially similar results hold if one consider a superposition of a finite number of Fock states. A similar analysis can be carried out for a coherent state $|\Psi\rangle=|\alpha\rangle$, which leads to the limit $|\alpha| \ll J / g_{0} \approx 10^{5}$. 


\section{EMITTER DECAY RATE IN THE THREE-CAVITY SYSTEM}

As mentioned in the main text, for the calculation in fig. 3b we assume a conservative value of $\Gamma / 2 \pi=0.05 \mathrm{GHz}$ for the rate of the emitter decay into the leaky modes (i.e., all the optical modes different from the three supermodes of the system). This value corresponds to the one measured for an NV center placed in a single defect cavity (similar to the one used here to realize a three-cavity system) and out of resonance with the optical mode [4]. However, significantly smaller radiative decay rates could in principle occur in the proposed structure, as we explain in the following. To evaluate the expected decay rate, we perform finite element method calculations (COMSOL) of the power emitted by an electric dipole (fig. S4) placed in either the target (blue line) or the left cavity (green line) of the structure considered in the main text. We then normalize the calculated powers by the power emitted by the same dipole in bulk diamond. In this way we obtain a prediction for the radiative decay rate of a $\mathrm{QE}$ (with the same position and polarization of the dipole) normalized to the radiative decay rate of the same QE in bulk. In agreement with the spatial patterns of the optical supermodes (see also sec. XII of this S.I.), a dipole placed in the lateral cavity can feed all the three optical supermodes, while one that is located in the target cavity does not emit into the mode $\hat{a}_{0}$. In particular, the calculation shows that the radiative decay rate of a $\mathrm{QE}$ placed in the cavity $\mathrm{T}$ is reduced by almost a factor $10^{3}$ with respect to the decay rate in bulk. As the measured lifetime of an NV center in bulk is about 12 ns [5], a radiative decay rate of the order of $\Gamma / 2 \pi=0.1 \mathrm{MHz}$ is in principle expected for the same emitter placed in the target cavity. We also note that the emission of a dipole in the target cavity is dominated by the coupling with the nonresonant coupled modes for the chosen set of parameters (see sec. VI), while the emission into leaky modes is expected to be even smaller. We therefore conclude that the assumed value of $50 \mathrm{MHz}$ is indeed a conservative, upper estimate of the $\mathrm{QE}$ decay rate.

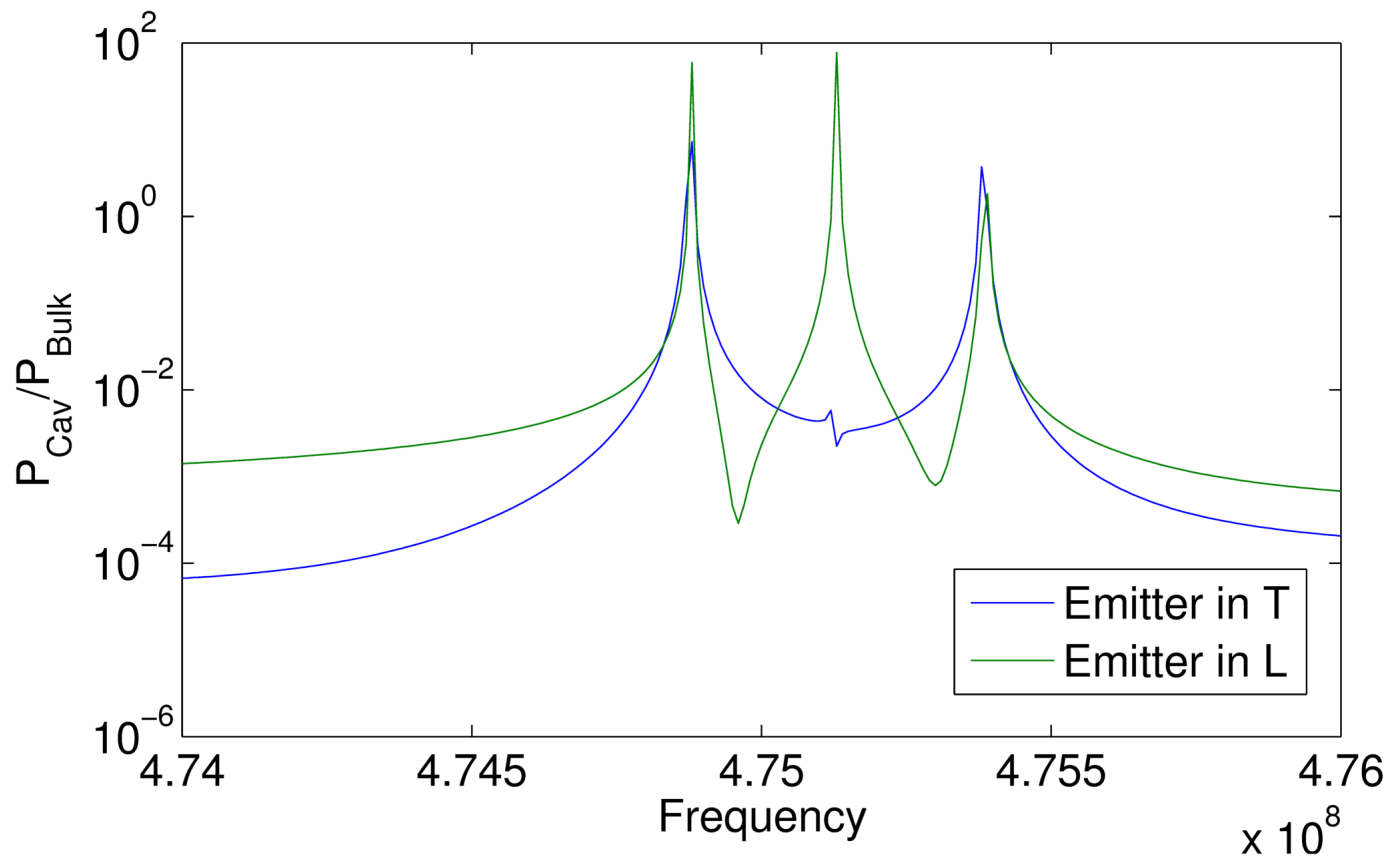

FIG. S4. Calculated power emitted by a dipole placed in the target (blue line) and left (green line) cavity of the three-cavity diamond structure discussed in the main text and in sec. XII of this SI. The powers $\left(P_{\mathrm{Cav}}\right)$ are normalized by the power emitted in bulk diamond $\left(P_{\mathrm{Bulk}}\right)$. 


\section{ADDITIONAL RADIATIVE LOSSES INTRODUCED BY THE SUPERMODES $\hat{a}_{+}$AND $\hat{a}_{-}$}

As shown in the main text, for a proper parameter choice the Hamiltonian of the threecavity system provides a tripartite interaction between the supermode $\hat{a}_{0}$, the QE and the mechanical mode. The interaction between the QE and the modes $\hat{a}_{ \pm}$is suppressed for large supermode separation $\left(\sqrt{2} J \gg \Omega_{\mathrm{M}}\right)$. However, these optical modes are still coupled with the emitter (since their fields at the emitter position is not zero), as shown in fig. S4. For large optical losses $\kappa>g$, the supermodes $\hat{a}_{ \pm}$are weakly coupled to the QE and therefore they introduce additional decay channels (denoted $\Gamma^{( \pm)}$). These additional decay channels become relevant when the other radiative decay channel $(\Gamma)$ is small and the optical linewidth is significant. This effect is important in understanding some features related to the fidelity of the created nonclassical states and to the ground-state cooling efficiency (see figs. 3(b,d) and accompanying discussion in the main text). $\Gamma^{( \pm)}$can be calculated as follows. If an optical mode with losses $\kappa$ is resonant with a QE and coupled with it at a rate $g^{\prime}<\kappa$, the $\mathrm{QE}$ decays into the optical mode at a rate $4 g^{\prime 2} / \kappa$. In the presence of a large spectral detuning $\delta \gg \kappa$, the decay rate is modified into $\frac{4 g^{\prime 2}}{\kappa} \cdot \frac{\kappa^{2} / 4}{\delta^{2}+\kappa^{2} / 4} \approx \frac{g^{\prime 2} \kappa}{\delta^{2}}$ [6]. In our case, $\delta=\sqrt{2} J$ and we replace $g^{\prime}=g / \sqrt{2}$, consistently with the notation in the main text where $g$ is the coupling between the QE and the uncoupled cavity. Therefore,

$$
\Gamma^{( \pm)}=\frac{g^{2} \kappa}{4 J^{2}}
$$

With respect to the QE-phonon swapping this additional decay channel is negligible when $\Gamma^{( \pm)} \ll \gamma \sqrt{n_{\text {cav }}} \Rightarrow \kappa \ll 2 J g_{0} \sqrt{n_{\text {cav }}} / g$, which corresponds to the vertical dashed-dotted line in fig. $3 \mathrm{c}$ of the main text.

\section{SELECTIVE PUMPING OF THE OPTICAL MODE OF INTEREST IN THE THREE-CAVITY SYSTEM}

In the three-cavity system the mode field coupling is due to a particular optical supermode $\left(\hat{a}_{0}\right)$, which, when the system is not perturbed by the mechanical displacement, features zero electric field in the central cavity and equal and opposite field amplitudes in the lateral cavities (see the first of eqs. S14 for $\Delta=0$ ). In order to enhance the mode field coupling it is therefore necessary to selectively pump this mode, without feeding the other two optical supermodes. This is possible by pumping the two lateral cavities at a frequency $\omega_{\mathrm{c}}$ with equal field amplitude $\mathcal{E}$ and opposite phases: excitation of modes $\hat{a}_{ \pm}$is then symmetry-forbidden. Focusing only on the optical part of the Hamiltonian, we have

$$
\hat{H}_{\text {opt }}=J\left[\hat{a}_{\mathrm{T}}^{\dagger}\left(\hat{a}_{\mathrm{R}}+\hat{a}_{\mathrm{L}}\right)+\text { h.c. }\right]+\mathcal{E}\left(\hat{a}_{R}^{\dagger}-\hat{a}_{L}^{\dagger}+\text { h.c. }\right)
$$

where we switched to a frame rotating at frequency $\omega_{\mathrm{c}}$. By writing the equations of motion for the three cavity field amplitudes, and assuming equal cavity losses $\kappa$, we can calculate the steady state population under this pumping scheme, namely $\bar{a}_{R}=-\bar{a}_{L}=2 \mathcal{E} / \kappa$ and $\bar{a}_{T}=0$. By comparing these results with eqs. S14 for $\Delta=0$, we see that only the mode $\hat{a}_{0}$ is fed, while the population of the other two modes remains strictly zero. Interestingly, in the limit of validity of the coupled mode theory, this result (i.e. the zero population of the supermodes $\hat{a}_{+}$and $\hat{a}_{-}$) is independent of $\kappa$. 


\section{THREE-CAVITY SYSTEM HAMILTONIAN AT LARGE DRIVING}

In the previous section we explained how the mode $\hat{a}_{0}$ can be selectively pumped. However, solving a full quantum model (eq. S13) with a large pumping of the optical cavities is computationally extremely challenging, because of the large Fock space dimensions required. We therefore solve a transformed version of the Hamiltonian in eq. S13, obtained through a displacement of the cavity operators. That is, we replace the operators by the sum of a steady-state amplitude and a fluctuating operator, i.e. $\hat{a}_{i}=\bar{a}_{i}+\delta \hat{a}_{i}$, where $i=\mathrm{L}, \mathrm{R}, \mathrm{T}$. The transformed Hamiltonian reads

$$
\begin{aligned}
\hat{H}_{\text {lin }}= & -\hat{\Delta} \delta \hat{a}_{L}^{\dagger} \delta \hat{a}_{\mathrm{L}}+\hat{\Delta} \delta \hat{a}_{R}^{\dagger} \delta \hat{a}_{\mathrm{R}}+\Omega_{\mathrm{M}} \hat{b}^{\dagger} \hat{b}+\frac{\omega_{\mathrm{A}}-\omega_{\mathrm{c}}}{2} \hat{\sigma}_{z}+J\left(\delta \hat{a}_{T}^{\dagger} \delta \hat{a}_{\mathrm{L}}+\text { h.c. }+\delta \hat{a}_{T}^{\dagger} \delta \hat{a}_{\mathrm{R}}+\text { h.c. }\right)+ \\
& +g\left(\delta \hat{a}_{\mathrm{T}} \hat{\sigma}_{+}+\text {h.c. }\right)+\frac{g_{0}}{\sqrt{2}} \sqrt{\frac{n_{\mathrm{cav}}}{2}}\left(\hat{b}^{\dagger}+\hat{b}\right)\left[\left(\delta \hat{a}_{L}^{\dagger}+\delta \hat{a}_{\mathrm{L}}\right)+\left(\delta \hat{a}_{R}^{\dagger}+\delta \hat{a}_{\mathrm{R}}\right)\right]
\end{aligned}
$$

where we defined $\sqrt{n_{\text {cav }}}=\sqrt{2}\left|\bar{a}_{R}\right|=\sqrt{2}\left|\bar{a}_{L}\right|$ and we have used the fact that, with the pumping scheme described in the previous section, $\bar{a}_{R}=-\bar{a}_{L}$ and $\bar{a}_{T}=0$. We note that this Hamiltonian is still equivalent to the original one, since no terms have been neglected. The Hamiltonian has been solved numerically (with the method discussed in sec. I) by either setting $n_{\text {cav }}=0$ (for the case in which the mode $\hat{a}_{0}$ is not externally pumped) or setting a fixed value of $n_{\text {cav }}$ (continuous optical pumping of the system). To address the case of a square-pulse excitation, we first calculate the time evolution of the (classical) cavity fields amplitudes upon the external pumping described above, neglecting the presence of the QE and the mechanical mode. From the amplitudes $\bar{a}_{R}(t)$ and $\bar{a}_{L}(t)$, we calculate the population of the mode $\hat{a}_{0}\left(n_{\text {cav }}(t)\right)$, which is used as a time-dependent parameter in solving the Hamiltonian in eq. S22.

\section{INFLUENCE OF CAVITY POPULATION AND DEPHASING ON THE FIDELITY OF THE ONE-PHONON STATE CREATION}
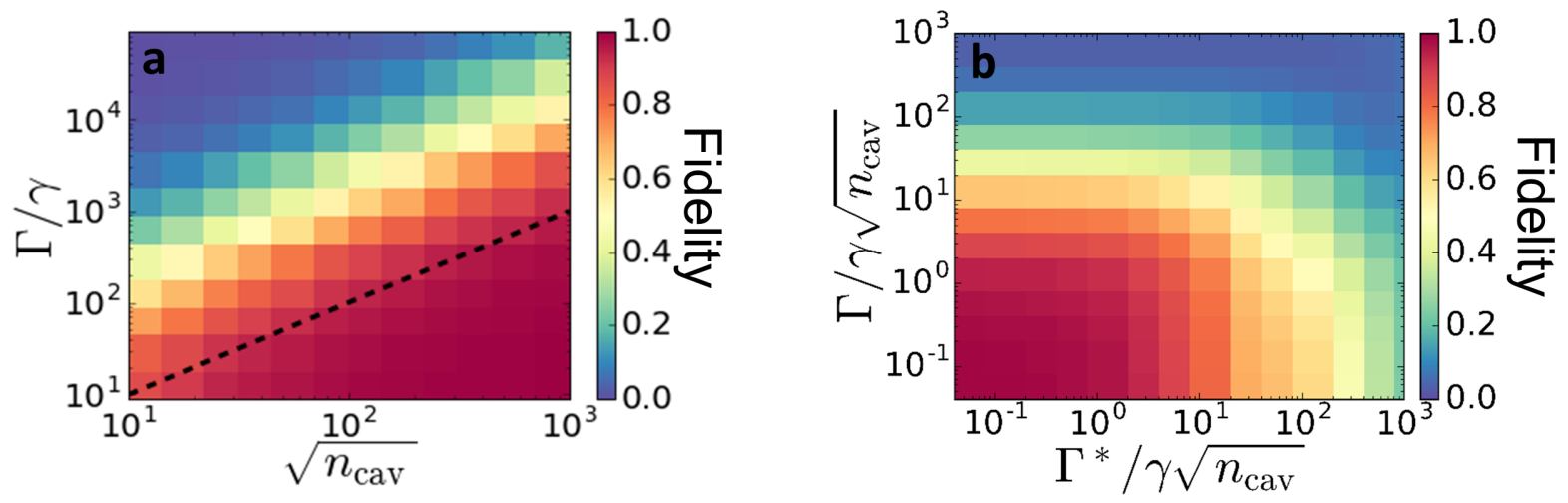

FIG. S5. (a) Fidelity of the creation of the one-phonon state versus the emitter decay rate $\Gamma$ and the population of the mode $\hat{a}_{0}\left(n_{\text {cav }}\right)$. The dashed black line indicates the condition $\Gamma / \gamma=\sqrt{n_{\text {cav }}}$. (b) Fidelity of the creation of the one-phonon state versus the emitter decay rate $\Gamma$ and the emitter pure dephasing rate $\Gamma^{*}$.

As mentioned in the main text, the mode field coupling rate can be enhanced by increasing the population of the mode $\hat{a}_{0}$. This is beneficial for, e.g., the fidelity of the emitter-phonon 
swapping. The colormap in fig. S5a shows the fidelity of the creation of the one-phonon state (calculated with the transformed Hamiltonian of the three-cavity system, eq. S22) versus the emitter decay rate $\Gamma$ and the population of the mode $\hat{a}_{0}$. All the other parameters are the same as in the main text. As expected, near-unity fidelity is obtained when $\gamma \sqrt{n_{\text {cav }}}>\Gamma$.

Due to the coherent nature of the MFC, pure dephasing of the QE is expected to further decrease the fidelity. As shown in fig. S5b, the QE decay $\Gamma$ and pure dephasing rates $\Gamma^{*}$ play a similar role in determining the fidelity, and $\Gamma^{*} \ll \gamma \sqrt{n_{\text {cav }}}$ is required as well to achieve large fidelity. We note that for the system considered for the calculations shown in this work (NV centers in diamond), the pure dephasing rate is typically of the same order of magnitude of the decay rate in bulk [7].

\section{CREATION OF OTHER NONCLASSICAL MECHANICAL STATES}

The MFC creates an interaction between a $\mathrm{QE}$ and a mechanical resonator whose coupling rate is controllable in time by the optical intensity. This unique characteristic allows us to create, in principle, any nonclassical state of the mechanical resonator, and the fidelity is only limited by the QE and optical losses. In general, as demonstrated by Law and Eberly [8], in any Jaynes-Cumming-like system in which the interaction between a quantum emitter and a bosonic field (i.e., the mechanics in this case) can be controlled in time, arbitrary quantum states of the bosonic field can be created. This is achieved by a sequence of pulses in which the QE is first partially excited from the outside, and then the excitation is transferred to the bosononic field by switching on the coupling [8].

In practice, simple nonclassical states such as larger Fock states or a superposition of Fock states can be easily realized by a sequence of few $\pi$-and/or $\pi / 2$-pulses applied to the QE and the cavity, as shown in fig. 3c of the main text. Here, we first explain the creation of these states within a simplified model in which the MFC is added ad-hoc to a system composed of one cavity, one mechanical resonator and a QE (eqs. 1-2 of the main text). Then, we show how the same dynamics, apart for the additional losses due to the other two optical modes, is obtainable with the three-cavity system. We used the same parameters as in the main text, $\left\{\omega_{\mathrm{c}}, \Omega_{\mathrm{M}}, g, g_{0}, J\right\}=2 \pi \cdot\left\{4.7 \cdot 10^{5}, 14,20,0.004,360\right\} \mathrm{GHz}$, and we consider the case of red-detuned cavity, $\omega_{\mathrm{A}}=\omega_{\mathrm{c}}+\Omega_{\mathrm{M}}$. The cavity losses are set to $\kappa /(2 \pi)=3 \mathrm{GHz}$. For simplicity, we neglect mechanical losses. The maximum photon population in the cavity mode upon external pumping is $\bar{n}_{\text {cav }}=5 \cdot 10^{4}$.

\section{X.1. One-cavity model with MFC}

We consider the simple Hamiltonian of eq. 2 (in which we already performed the linearisation of the optical field). Moreover, we add an external pumping to the atom, we consider only the dominant terms of the tripartite interaction (see discussion in the main text after eq. 1) and we switch to a reference frame rotating at frequency $\Omega_{\mathrm{M}}=\omega_{\mathrm{A}}-\omega_{\mathrm{c}}$. The Hamiltonian reads

$$
\hat{H}=\gamma \sqrt{n_{\mathrm{cav}}(t)}\left(\hat{b} \hat{\sigma}_{+}+\hat{b}^{\dagger} \hat{\sigma}_{-}\right)+E_{A}(t)\left(\hat{\sigma}_{+}+\hat{\sigma}_{-}\right)
$$

where $E_{A}(t)$ is a time-dependent external field resonant with the QE and $n_{\text {cav }}(t)$ is the population of the optical mode. Similar to the calculations shown before, $n_{\text {cav }}(t)$ is calculated as the response of the cavity mode to a sequence of square pulses. The value of $\gamma$ is assumed identical to the one obtainable in the three-cavity system with the same set of parameters, i.e., $\gamma=g g_{0} /(2 J)=(2 \pi) \cdot 0.11 \mathrm{MHz}$. 

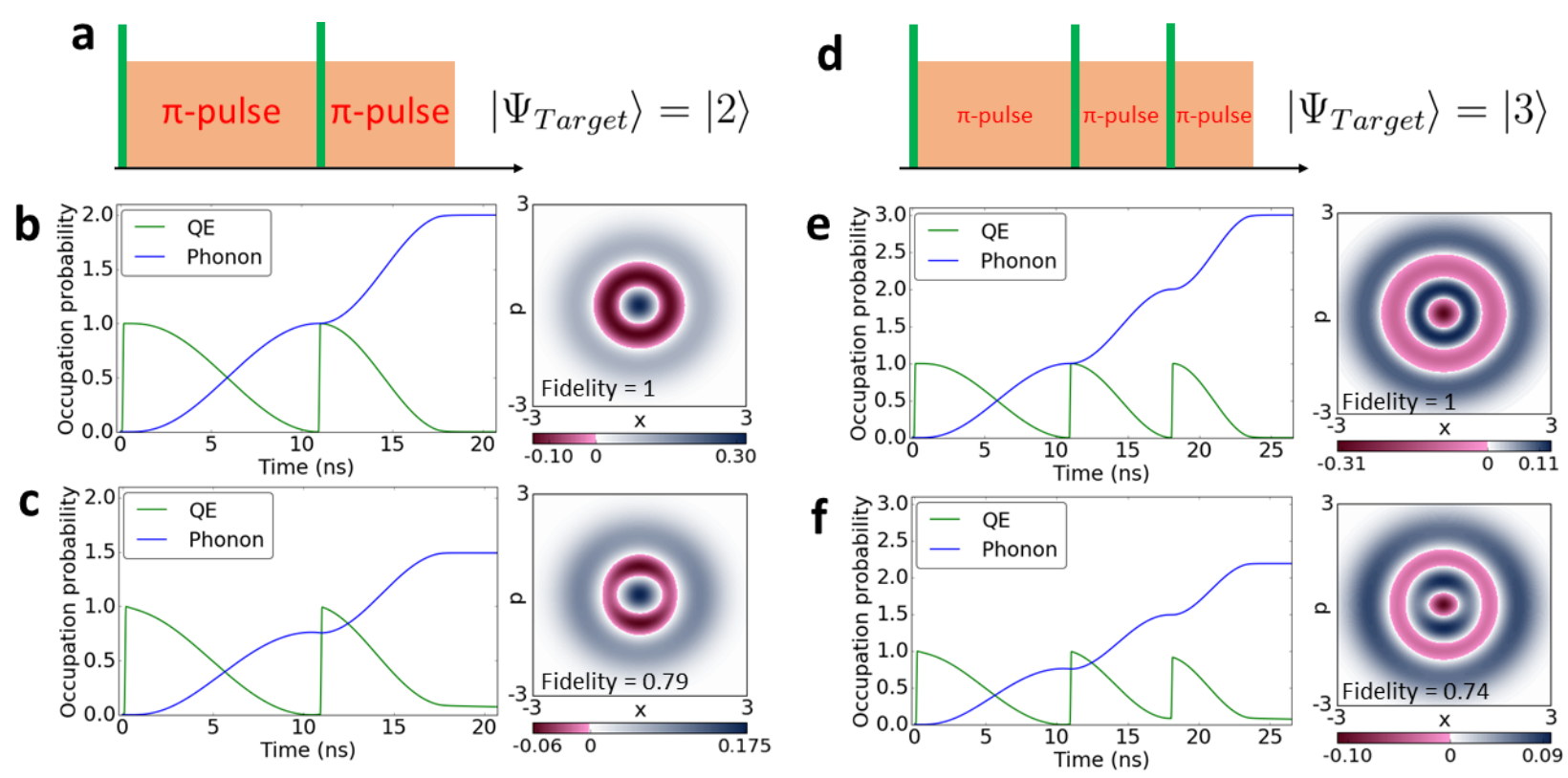

FIG. S6. Creation of larger Fock states with the one-cavity MFC model. (a-c) Creation of the Fock state $n=2$. (a) Protocol used. Green rectangles indicate $\pi$-pulses applied to the QE, while red rectangles are pulses applied to the cavity. (b) Time evolution in absence of QE losses (left) and Wigner map of the mechanical state created (right). (c) Same as in (b), but with finite QE losses $\Gamma /(2 \pi)=0.05 \mathrm{GHz}$. (d-f) Same as in (a-c), for the Fock state $n=3$.

Arbitrary mechanical Fock states can be obtained by first exciting the QE and then transferring the excitation to the mechanical resonator by exciting the cavity mode with a $\pi$-pulse, i.e., a pulse with a duration $\Delta t$ and a maximum photon population $\bar{n}_{\text {cav }}$ such that $(2 \gamma \sqrt{m}) \sqrt{\bar{n}_{\text {cav }}} \Delta t=\pi$, where $m$ is the phonon number to be achieved at the end of the pulse. In fig. S6 we show the protocols to create the Fock states $n=2$ (fig. S6a) and $n=3$ (fig. S6d). We calculated the system dynamics upon these pumping protocols for both the lossless case (figs. S6b and S6e) and for the case in which the QE has finite losses identical to the case considered in the main text, $\Gamma /(2 \pi)=0.05 \mathrm{GHz}$ (figs. S6c and S6f). For each case we show the temporal evolution of the system (in terms of QE and phonon occupation probability) and the Wigner map of the created mechanical states. The fidelity of the created states are displayed inside the corresponding Wigner maps. For zero emitter losses, the desired nonclassical states are created with unity fidelity, while the fidelity is somewhat reduced when QE losses are introduced. Nonetheless, it is high enough to display the characteristic negativity (nonclassicality) of the Wigner function.

Other nonclassical states, such as the superposition of two or more Fock states, can be obtained by using different pulse sequences. For example, if in the case of the Fock state $n=2$ (fig. S6a) the first $\pi$-pulse on the cavity is replaced by a $\pi / 2$ pulse, the state $(|g, 0\rangle-|g, 2\rangle) / \sqrt{2}$ is obtained (fig. S7a), where $g$ refers to the ground state of the QE and the numbers are the Fock states of the mechanics. The temporal evolution of the system and the Wigner map of the mechanical states are shown in figs. S7b and S7c, for the lossless case and the case with finite QE losses, respectively. Again, the only limitation to the fidelity of the created state is due to the QE losses. 


\section{X.2. Three-cavity model}

We now verify that these nonclassical states can be obtained also in the three-cavity system with a large optical pumping. We numerically solve the full, non-linearized, Hamiltonian in eq. S22 (with an additional term to externally pump the QE), in which $n_{\text {cav }}$ is a time-dependent parameter which describes the optical pumping of the mode $\hat{a}_{0}$. In figs.

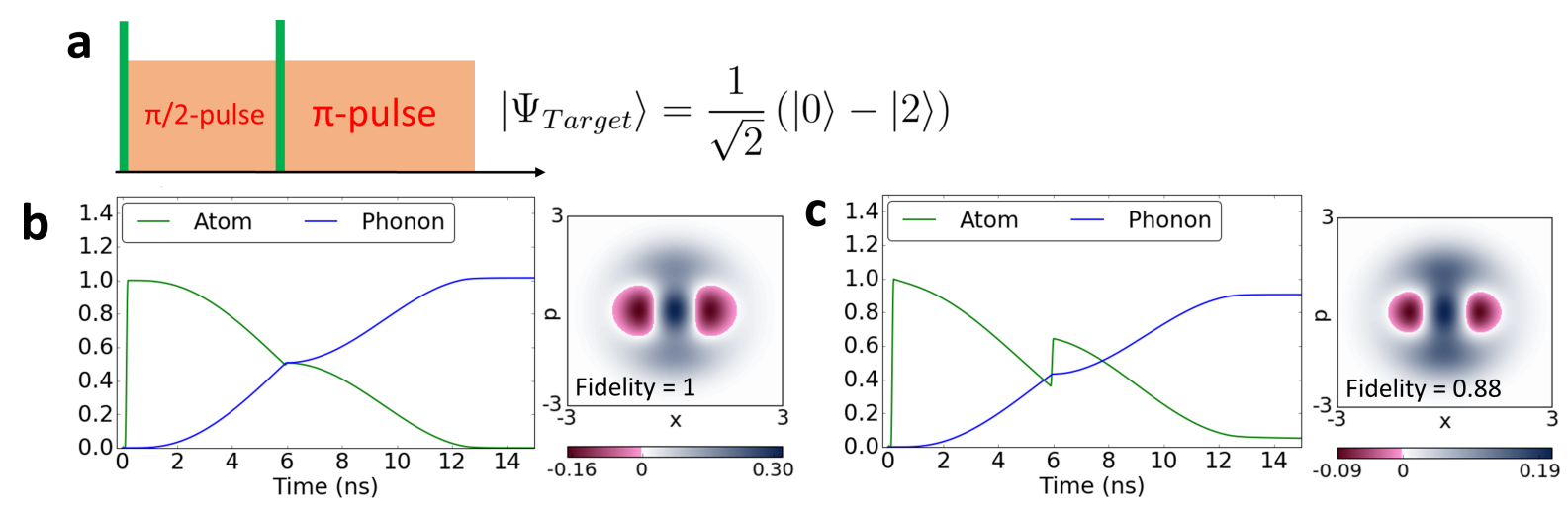

FIG. S7. Creation of the superposition state $\left|\Psi_{\text {Target }}\right\rangle=(|g, 0\rangle-|g, 2\rangle) / \sqrt{2}$ with the one-cavity MFC model. (a) Protocol used. Green rectangles indicate $\pi$-pulses applied to the QE, while red rectangles are pulses applied to the cavity. (b) Time evolution in absence of QE losses (left) and Wigner map of the mechanical state created (right). (c) Same as in (b), but with QE losses $\Gamma /(2 \pi)$ $=0.05 \mathrm{GHz}$.

$$
\left|\Psi_{\text {Target }}\right\rangle=|2\rangle
$$
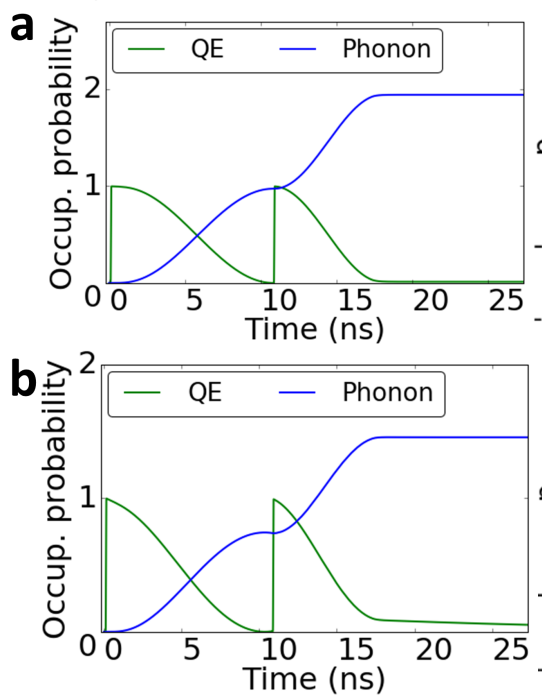
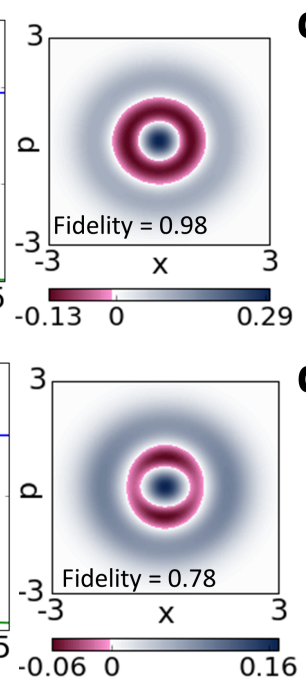
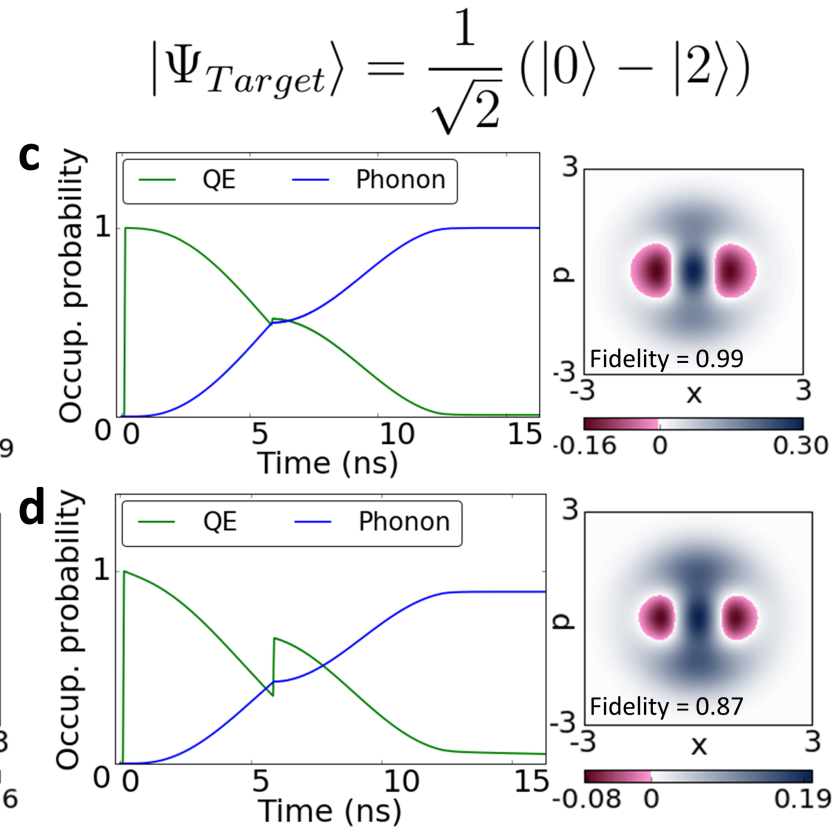

FIG. S8. Creation of nonclassical mechanical states with the three-cavity system. (a-b) Creation of the Fock state $n=2$ (same pumping protocols as fig. S6a). (a) Time evolution in absence of QE losses (left) and Wigner map of the mechanical state created (right). (b) Same as in (a), but with QE losses $\Gamma /(2 \pi)=0.05 \mathrm{GHz}$. (c-d) Creation of the superposition state $\left|\Psi_{\text {Target }}\right\rangle=$ $(|g, 0\rangle-|g, 2\rangle) / \sqrt{2}$ (same pumping protocols as fig. S7a). Panels (c) and (d) show the same plots as in (a-b), but for the superposition state. 
S8 $(\mathrm{a}-\mathrm{b})$ we show the creation of the Fock state $n=2$, without (panel a) and with (panel b) emitter losses $(\Gamma /(2 \pi)=0.05 \mathrm{GHz})$. The pumping protocol used is the same as in fig. S6a. The only difference with respect to the one-cavity system is that, even for zero QE losses, the fidelity (0.98) is lower than one, as a result of the coupling of the QE with the other two optical supermodes. Similar results are obtained in the creation of the superposition state $\left|\Psi_{\text {Target }}\right\rangle=(|g, 0\rangle-|g, 2\rangle) / \sqrt{2}$ (figs. S8(c-d)) that, in absence of QE losses (fig. S8c), is created with fidelity 0.99 . While reducing the cavity losses $(\kappa)$ will reduce the QE decay induced by the other two optical supermodes $\left(\Gamma^{( \pm)}\right.$, see sec. VI), it will also increase the temporal duration of the cavity response to the external pumping. This results in a longer duration of the excitation transfer between the $\mathrm{QE}$ and the phonon, and therefore the $\mathrm{QE}$ decay $(\Gamma)$ will have a larger influence on the fidelity. This trade-off leads to an optimal value of $\kappa$ that maximizes the fidelity, which is numerically found to be $\kappa_{\text {opt }} /(2 \pi) \approx 3 \mathrm{GHz}$ in this case.

\section{ROLE OF THE OTHER TWO MECHANICAL SUPERMODES}

In the main text, and in the previous sections of this supplemental information, we assumed that only the mechanical supermode of interest is relevant for the system dynamics. To verify this assumption, we performed additional numerical calculations based on the Hamiltonian in eq. S10, i.e. by considering explicitly the three mechanical uncoupled modes.
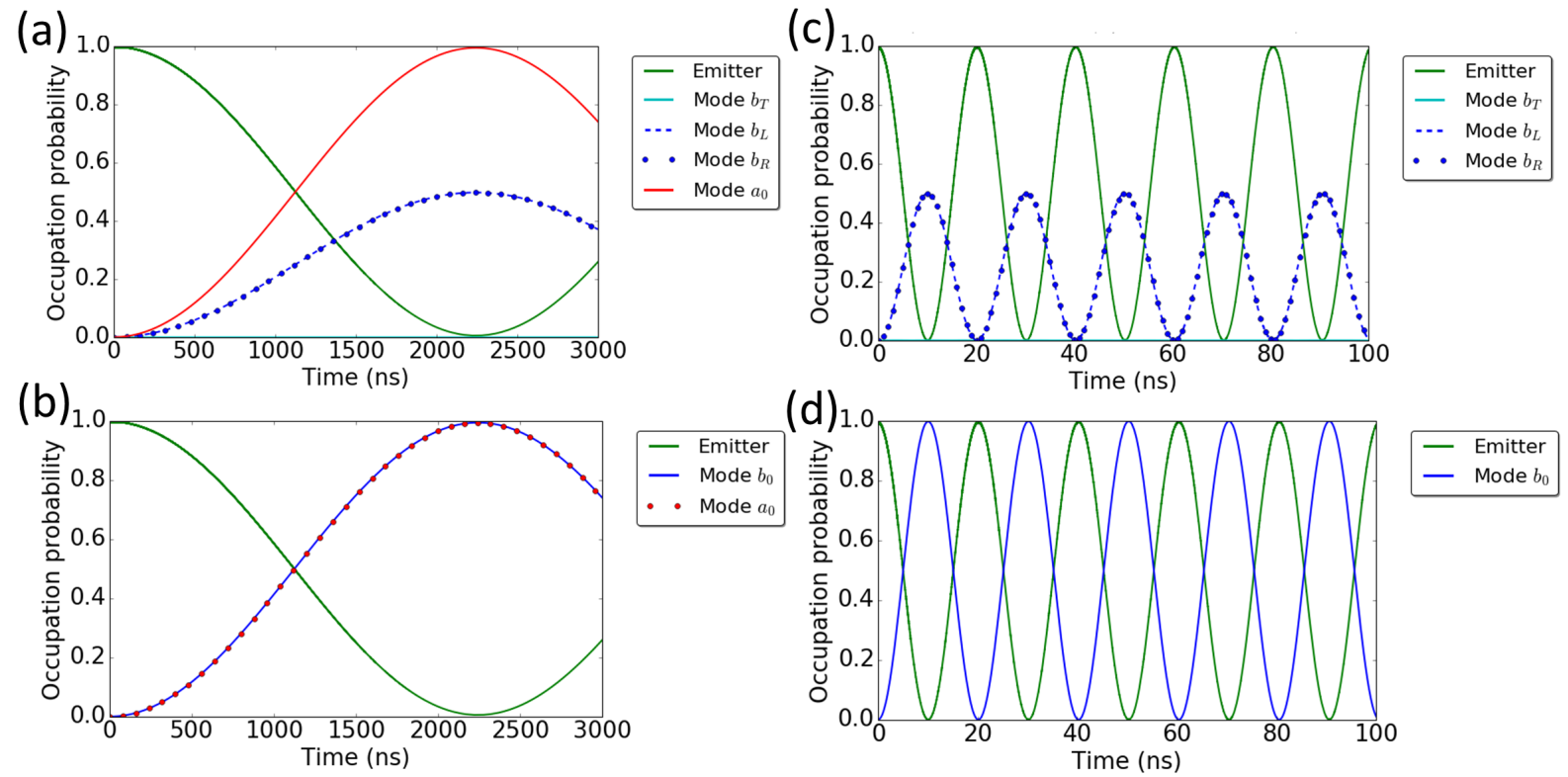

FIG. S9. Numerical calculation of the full Hamiltonian in eq. S10, i.e. considering all mechanical modes. The parameters of the system are the same as in fig. 3a of the main text. (a-b) Vacuum oscillations of the system (i.e. the optical cavities are not pumped). (a) The mechanical modes $\hat{b}_{\mathrm{L}}$ and $\hat{b}_{\mathrm{R}}$ are equally populated (blue solid and blue dotted lines) and reach a maximum occupation probability of 0.5 , while the mechanical mode $\hat{b}_{\mathrm{T}}$ is not populated (cyan solid line). (b) When switching to the supermode basis (through eqs. S11), the mode $\hat{b}_{0}$ behaves exactly as shown in previous calculations in which the other supermodes were neglected. (c-d) Same as in panels (a-b) but with an external pumping of the optical cavities, such that the optical supermode $\hat{a}_{0}$ contains $n=5 \cdot 10^{4}$ photons. 
We use the same parameters as in the main text, and a mechanical coupling $J_{\mathrm{M}} /(2 \pi)=50$ $\mathrm{MHz}$. Initially, we assume no mechanical losses $\left(\Gamma_{\mathrm{M}}=0\right)$. After calculating the time evolution of the modes $\hat{b}_{\mathrm{L}}, \hat{b}_{\mathrm{R}}$ and $\hat{b}_{\mathrm{T}}$, we derive the time evolution of the three mechanical supermodes based on eqs. S11. As shown in fig. S9, both in the unpumped and the pumped case the dynamics of the mechanical mode $\hat{b}_{0}$ matches that found previously, where the other mechanical modes were neglected. The population of the other two mechanical supermodes $\left(\hat{b}_{+}\right.$and $\left.\hat{b}_{-}\right)$is always zero (not shown in these plots). This is due to the fact that these other mechanical supermodes interact with the optical cavities in a way which does not lead to any tripartite interaction (see eq. S12), and their presence is therefore negligible for proper choice of frequencies.

Interestingly, these results hold true even for large mechanical losses $\Gamma_{\mathrm{M}} \gg J_{\mathrm{M}}$, and the fidelity of the one-phonon-state creation does not depend on the mechanical interaction $J_{\mathrm{M}}$, as we show in fig. S10a. Decrease of the fidelity is only observed if the mechanical losses become larger than $\gamma \sqrt{n_{\text {cav }}}$. The fact that the the presence of the other two mechanical supermodes can be completely neglected is also confirmed by fig. S10b: here, we compare the phonon population of the mode $\hat{b}_{0}$ versus $\Gamma_{\mathrm{M}}$ for the case of a three-cavity system (i.e. a horizontal cut of panel a) with the same graph calculated for a system composed by one optical cavity and one mechanical resonator, where the MFC is introduced ad-hoc (eq. 2 of the main text). The identical behaviours indicate that a large $\Gamma_{\mathrm{M}}$ introduces only additional losses but does not make the system interact with the other mechanical supermodes. The small deviation between the two curves for $\Gamma_{\mathrm{M}} /\left(\gamma \sqrt{n_{\text {cav }}}\right)<1$ is due to the additional losses present in the three-cavity system due to the optical modes $\hat{a}_{ \pm}$.

From a fundamental point of view, therefore, there are no constraints on the mechanical coupling $J_{M}$. Nonetheless, for practical reasons it could be beneficial to work in a regime where $J_{\mathrm{M}}>\Gamma_{\mathrm{M}}$, such that the mode of interest is spectrally separated from the other mechanical supermodes and it can be individually addressed in other ways for, e.g., cooling or readout.
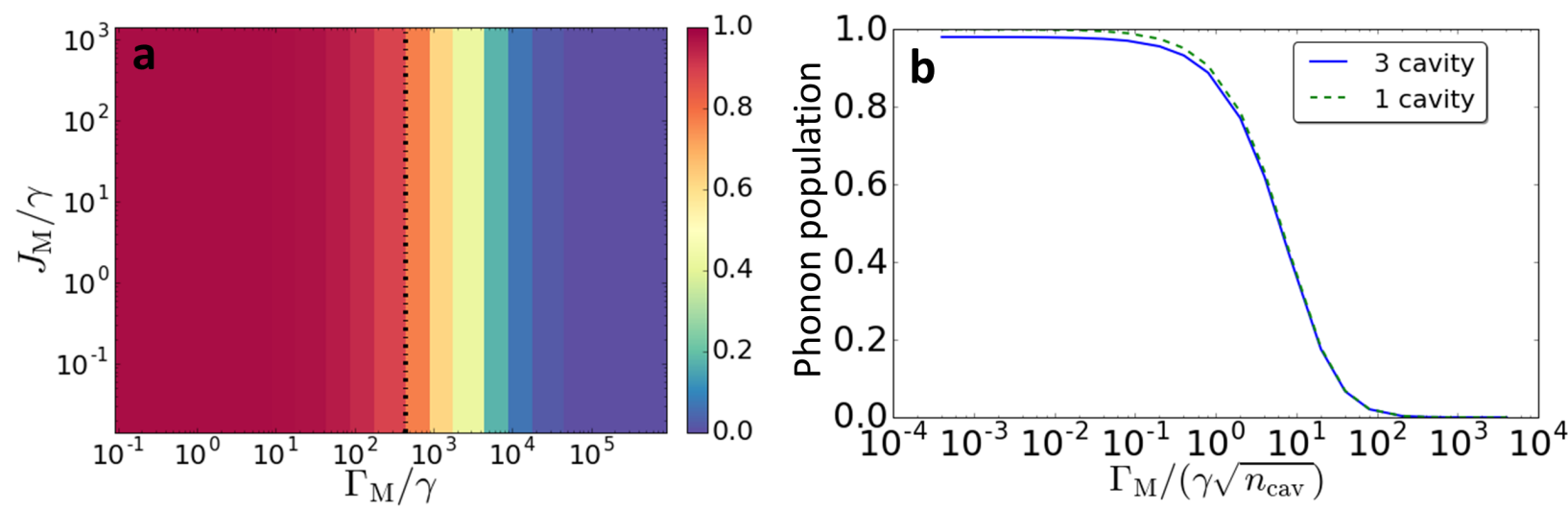

FIG. S10. Calculations of three-cavity system without neglecting the other mechanical supermodes. The optical mode $\hat{a}_{0}$ is continuosly pumped with $n_{\text {cav }}=5 \cdot 10^{4}$ and the QE is initially excited. All the other parameters are the same as in the main text and the QE losses have been neglected for simplicity. (a) Maximum phonon population (of the mode $\hat{b}_{0}$ ) achieved during the sysstem evolution, versus the mechanical interaction $J_{\mathrm{M}}$ and the mechanical losses $\Gamma_{\mathrm{M}}$. The dashed-dotted vertical line indicates the condition $\Gamma_{\mathrm{M}}=\gamma \sqrt{n_{\text {cav }}}$. (b) Horizontal cut of panel (a) for the lowest value of $J_{\mathrm{M}}$ (solid blue line) compared with the same quantity calculated in a MFC model with one cavity and one resonator (green dashed line, see eq. 2 of main text). 


\section{DESIGN OF THE PROPOSED STRUCTURE}

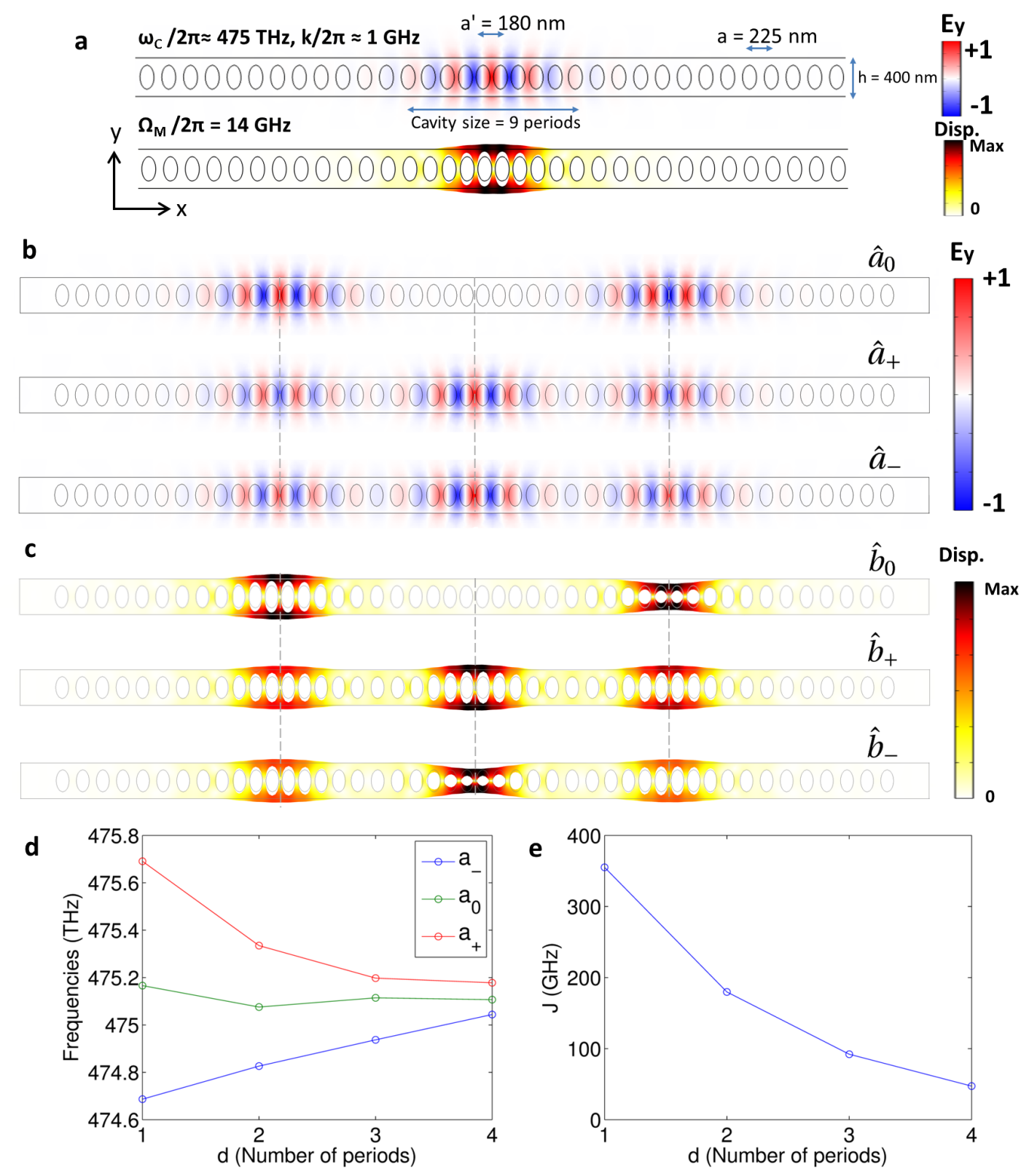

FIG. S11. Numerical investigation of the proposed structure (see text for details). (a) Electric field pattern (top) and mechanical displacement pattern (bottom) of the colocalized optical and mechanical mode for a single cavity. The $y$-component of the electric field is shown. The mode frequencies and the optical losses are indicated in the figure. (b-c) Three identical cavities on a nanobeam, separated by 2 periods. The position of the three cavities is marked by the vertical grey dashed lines. (b) Electric field pattern of the three optical supermodes when the mechanical mode is at rest. (c) Displacement pattern of the three mechanical supermodes. (d) Frequencies of the three optical supermodes as a function of the cavities separation (i.e. number of periods). (e) Values of the optical interaction $J$ (extrapolated from the plot in panel (d), see text) versus the cavity separation.

The structure proposed in the main text is composed of three in-line defect cavities in a 
photonic crystal diamond nanobeam. Simulations have been performed with a finite element method commercial software (COMSOL). We first designed the single cavity structure (fig. S11a), which is realized by quadratically tapering the lattice constant across 9 lattice periods, from the unperturbed value of $a=225 \mathrm{~nm}$ to the central value of $a^{\prime}=0.8 a=180 \mathrm{~nm}$. The design is inspired by the one proposed recently by Lee et al.[4]. The holes have elliptical shape, with semiaxes equal to $a_{x}=0.32 a$ and $a_{y}=0.55 a$. The width of the nanobeam (along the $y$-direction) is $w=400 \mathrm{~nm}$, and the thickness is $200 \mathrm{~nm}$. This structure supports an optical mode with frequency $\omega_{\mathrm{c}} / 2 \pi=475 \mathrm{THz}$ and $Q \approx 3 \cdot 10^{5}$ (fig. S11a, top panel), and a co-localized mechanical mode with frequency $\Omega_{\mathrm{M}} / 2 \pi=14 \mathrm{GHz}$ (fig. S11a, bottom panel). The dispersive optomechanical coupling between the optical and mechanical modes has been calculated by evaluating the moving-boundary and photoelastic contribution separately (as discussed in ref. 9) and amounts to $g_{0} / 2 \pi \approx 4 \mathrm{MHz}$. We notice that we did not perform any systematic optimization on our design, and therefore the optical Q factor and optomechanical coupling rate could be further increased by carefully optimizing the design parameters (e.g., by tapering also the holes' semiaxes) as discussed by other authors [9].

After having chosen a design for the single cavity, we considered three identical defect cavities on the same nanobeam, separated by an equal distance $d$, defined as the number of unperturbed periods between two adjacent cavities. For each distance $d$, we calculated the optical and mechanical supermodes. Figure S11b shows the $E_{y}$ field component for the three optical supermodes. Note that the optical mode of interest, $\hat{a}_{0}$, has no field in the central cavity, differently from the other two modes. The modes $\hat{a}_{+}$and $\hat{a}_{-}$differ in the relative sign between the field in the lateral cavities and the field in the central cavity, as expected from the results of the coupled-mode theory (eq. S14). Figure S11c shows the displacement pattern of three mechanical supermodes. Similarly to the optical case, the mechanical supermodes $\hat{b}_{+}$and $\hat{b}_{-}$have opposing oscillation phase in the central and the lateral cavities. Figure S11d shows the frequencies of the three optical supermodes versus the cavity separation $d$. The frequency of the mode $\hat{a}_{0}$ is expected to be independent of the cavity interaction $J$ (and therefore the cavity distances). The small frequency deviations observed can be due to either the finite mesh size (which makes the cavities slightly different from each other) or to the breakdown of the coupled-mode theory for very short cavity distances. We notice that this does not affect our theoretical model, since the mechanical movement does not change the rate $J$. In fig. S11e we show the estimated optical interaction rate $J$ as a function of the inter-cavity distance. For each distance $d, J$ has been estimated by the formula $\left|\omega_{+}-\omega_{-}\right|=2 \sqrt{2} J$.

In fig. 2I of the main text we show the expected pattern of the mode $\hat{a}_{0}$ for the case in which the system is mechanically perturbed, such that the lateral cavities are detuned by $\Delta / J=0.5$. The shown electric field pattern has been calculated analytically from the formula

$$
E_{0, \Delta}(x, y)=C_{0}(\Delta) E_{0}(x, y)+C_{+}(\Delta) E_{+}(x, y)+C_{-}(\Delta) E_{-}(x, y),
$$

where $E_{i}(x, y)$, with $i=\{0,+,-\}$, are the electric field patterns of the supermodes when the mechanical mode is at rest (fig. S11b), and the coefficients $C_{i}(\Delta)$ are calculated analytical from the coupled mode theory.

\section{TRIPARTITE INTERACTION IN A FABRY-PEROT CAVITY}

The interaction described in this work relies on a variation of the electric field at the emitter position upon a mechanical displacement. A small field variation occurs in principle also in a simple Fabry-Perot (FP) cavity when one of the mirrors is displaced by a mechanical 
resonator [10-12]. However, the interaction strength in this case is much smaller than the one obtained in the two and three cavity systems. In particular, for an emitter placed in a node of the $n$-th FP mode, the tripartite coupling rate reads $\gamma_{n}=\pi g g_{0} / \omega_{1}$, where $g$ is the maximum Rabi coupling between the QE and the $n$-th mode, $g_{0}$ is the dispersive coupling induced by the mirror movement and $\omega_{1}$ is the fundamental cavity frequency. The coupling rate bears similarity with the one derived for the two- and three-cavity systems, but with the important difference that the role of the intercavity interaction rate $J$ is taken by $\omega_{1}$, which, in the FP cavity, corresponds also to the frequency spacing between the unperturbed modes. This, in the visible and near-IR regime, severely limits the achievable values of $\gamma_{n}$, especially since any effort to reduce $\omega_{1}$ reduces both $g$ and $g_{0}$.

[1] J. Johansson, P. Nation, and F. Nori, Comput. Phys. Commun. 183, 1760 (2012).

[2] M. A. Nielsen and I. L. Chuang, Cambridge: Cambridge University Press 2, 23 (2000).

[3] M. Ludwig, A. H. Safavi-Naeini, O. Painter, and F. Marquardt, Phys. Rev. Lett. 109, 063601 (2012).

[4] J. C. Lee, D. O. Bracher, S. Cui, K. Ohno, C. A. McLellan, X. Zhang, P. Andrich, B. Alemán, K. J. Russell, A. P. Magyar, I. Aharonovich, A. B. Jayich, D. Awschalom, and E. L. Hu, Appl. Phys. Lett. 105, 261101 (2014).

[5] A. Faraon, C. Santori, Z. Huang, V. M. Acosta, and R. G. Beausoleil, Phys. Rev. Lett. 109, $033604(2012)$

[6] P. Lodahl, S. Mahmoodian, and S. Stobbe, Rev. Mod. Phys. 87, 347 (2015).

[7] L. Robledo, H. Bernien, I. van Weperen, and R. Hanson, Phys. Rev. Lett. 105, 177403 (2010).

[8] C. Law and J. Eberly, Phys. Rev. Lett. 76, 1055 (1996).

[9] M. J. Burek, J. D. Cohen, S. M. Meenehan, T. Ruelle, S. Meesala, J. Rochman, H. A. Atikian, M. Markham, D. J. Twitchen, M. D. Lukin, O. Painter, and M. Lončar, Optica 3, 1404 (2016).

[10] S. Barzanjeh, M. H. Naderi, and M. Soltanolkotabi, Phys. Rev. A 84, 063850 (2011).

[11] Y. Chang, H. Ian, and C. Sun, J. Phys. B 42, 215502 (2009).

[12] W. Wang, L. Wang, and H. Sun, J. Korean Phys. Soc. 57, 704 (2010). 